\title{
A Two-Phenotype Model of Immune Evasion by Cancer Cells
}

Citation for published version (APA):

Bayer, P., Brown, J., \& Stankova, K. (2017). A Two-Phenotype Model of Immune Evasion by Cancer Cells. Maastricht University, Graduate School of Business and Economics. GSBE Research Memoranda No. 029 https://doi.org/10.26481/umagsb.2017029

Document status and date:

Published: 21/11/2017

DOI:

10.26481/umagsb.2017029

Document Version:

Publisher's PDF, also known as Version of record

\section{Please check the document version of this publication:}

- A submitted manuscript is the version of the article upon submission and before peer-review. There can be important differences between the submitted version and the official published version of record.

People interested in the research are advised to contact the author for the final version of the publication, or visit the DOI to the publisher's website.

- The final author version and the galley proof are versions of the publication after peer review.

- The final published version features the final layout of the paper including the volume, issue and page numbers.

Link to publication

\footnotetext{
General rights rights.

- You may freely distribute the URL identifying the publication in the public portal. please follow below link for the End User Agreement:

www.umlib.nl/taverne-license

Take down policy

If you believe that this document breaches copyright please contact us at:

repository@maastrichtuniversity.nl

providing details and we will investigate your claim.
}

Copyright and moral rights for the publications made accessible in the public portal are retained by the authors and/or other copyright owners and it is a condition of accessing publications that users recognise and abide by the legal requirements associated with these

- Users may download and print one copy of any publication from the public portal for the purpose of private study or research.

- You may not further distribute the material or use it for any profit-making activity or commercial gain

If the publication is distributed under the terms of Article $25 \mathrm{fa}$ of the Dutch Copyright Act, indicated by the "Taverne" license above, 


\section{Maastricht University}

Péter Bayer, Joel S. Brown, Katerina Stankova

A Two-Phenotype Model of Immune Evasion by Cancer Cells

$\mathrm{RM} / 17 / 029$

\section{GSBE}

Maastricht University School of Business and Economics

Graduate School of Business and Economics

P.O Box 616

NL- 6200 MD Maastricht

The Netherlands 


\title{
A Two-Phenotype Model of Immune Evasion by Cancer Cells*
}

\author{
Péter Bayer ${ }^{\dagger} \quad$ Joel S. Brown ${ }^{\ddagger}$ \\ Kateřina Staňkovás
}

November 10, 2017

\begin{abstract}
We propose a model with two types of cancer cells differentiated by their defense mechanisms against the immune system. "Selfish" cancer cells develop defense mechanisms that benefit the individual cell, whereas "cooperative" cells deploy countermeasures that increase the chance of survival of every cell. Our phenotypes capture the two main features of the tumor's efforts to avoid immune destruction, crypticity against immune cells for the selfish cells, and tumor-induced immunosuppression for the cooperative cells. We identify steady states of the system and show that only homogeneous tumors can be stable in both size and composition. We show that under generic parameter values, a tumor of selfish cells is more benign than a tumor of cooperative cells, and that a treatment against cancer crypticity may promote immunosuppression and increase cancer growth.
\end{abstract}

Keywords: Cancer heterogeneity, cancer ecology and evolution, immunoediting, immunosuppression, immunotherapy.

*This work is sponsored by the European Union's Horizon 2020 research and innovation program under the Marie Skłodowska-Curie grant agreement No 690817, National Institute of Health grants U54CA143970-1 and RO1CA170595, and a grant from the James S. McDonnell Foundation. Bayer received financial support from the Centre for Human Enhancement and Learning. We thank Jessica Cunningham, Jean-Jacques Herings, Abdel Halloway, Ronald Peeters, Frank Thuijsman, Christopher Whelan, Li You, audiences of the 2nd 4C Modelling Workshop at the City University of London, the MLSE Seminar at Maastricht University, participants of the biology lab meetings at the University of Illinois at Chicago, and the members of the Integrated Mathematical Oncology Department at Moffitt for valuable feedback and suggestions. Any errors are our own.

${ }^{\dagger}$ Corresponding author. Department of Economics, Maastricht University, P.O. Box 616, 6200 MD Maastricht, The Netherlands. E-mail: p.bayer@maastrichtuniversity.nl

$\ddagger$ University of Illinois at Chicago, Department of Biological Sciences. 845 West Taylor Street Chicago, IL 60607, United States and Department of Integrated Mathematical Oncology, Moffitt Cancer Center, 12902 USF Magnolia Drive, Tampa, FL 33612, United States. E-mail: Joel.Brown@moffitt.org

$\S$ Department of Data Science \& Knowledge Engineering, Maastricht University, P.O. Box 616, 6200 MD Maastricht, The Netherlands and Delft Institute of Applied Mathematics, Delft University, Mekelweg 4, 2628 CD Delft, The Netherlands. E-mail: k.stankova@maastrichtuniversity.nl. 


\section{Introduction}

The immune system influences cancer initiation and progression. One of the hallmarks of cancer is evasion of the immune system (Hanahan and Weinberg, 2011). The immune system is how multi-cellular organisms mount an adaptive response to diseases and pathogens (including cancer). There are innate and adaptive mechanisms of immunity. Innate immunity provides a relatively undirected but permanent defense against pathogens using a variety of white blood cells that include phagocytes and natural killer cells. Dendritic cells (a subset of phagocytes) provide a link between the innate and adaptive immune system. Upon encountering foreign proteins or molecules (antigens) the dendritic cells can entrain T-lymphocytes (T-cells) by modifying their receptor proteins to recognize the antigen. As part of the adaptive immune system, the killer T-cells now possess receptors that will recognize the surface antigen on nucleated cells such as infectious protozoans, viral infected normal cells, and even cancerous cells. Upon making contact, the T-cell is able to breach the target cell's membrane and introduce lethal cytotoxins.

When treating cancer, a high immune system infiltration into a tumor often begets a positive prognosis (Parcesepe et al., 2016) as immune cells inhibit the growth and spread of the tumor. Immunotherapy tries to trigger an effective immune response to the cancer (Dimberu and Leonhardt, 2011). Such therapies may introduce retroviruses into the tumor. The retroviruses present antigens that induce an immune response. Or, the patient's own immune system may be boosted by entraining T-cells on cancer cells drawn from the patient (Morgan et al., 2006). The antigens and entrained killer T-cells are then injected into the patient as a form of 'vaccine' to create a more directed and effective immune response to the cancer cells within the tumor. Finally, checkpoint therapies directly target the immune evasion traits of cancer cells (Goswami et al., 2016) making it easier for T-cells to encounter and attack cancer cells.

The adaptive immune response to cancer cells via T-cell activation has been conceptualized and modeled as a competition between killer T-cells (and associated macrophages, helper-cells, neutrophils, etc.) and the cancer cells. Eftimie et al. (2011) provide an extensive review of common spatially homogeneous mathematical models describing the interactions between a malignant tumor and the immune system, starting from the single equation models for tumor growth and adding complexity. Nani and Freedman (2000) construct a model of competition between normal cell and cancer cells during the process of immunotherapy. Robertson-Tessi et al. (2012) and Nakada et al. (2016) focus on the components of the immune system. Our model complements the literature by focusing on the various strategies available to the cancer cells in the game played by the cancer cells and the immune system. 
Unfortunately for the patient, cancer cells evolve resistance to the immune system and to immunotherapies (Ribas, 2015; Sharma et al., 2017). A number of papers have used agent based models to examine the evolution of resistance to various types of treatment or drugs by imagining two populations of cancer cells - one susceptible to treatment, the other not or less so (Panetta, 1998; Tomasetti and Levy, 2010; Sun et al., 2016). Other models see resistance as the cancer cells outcompeting the immune cells (De Pillis and Radunskaya, 2001). Baar et al. (2016) build a stochastic agent-based model where resistant phenotypes and genotypes are selected via mutation as they interact with a heterogeneous population of T-cells.

We consider an evolutionary game between two types of cancers cells in response to the immune system (Dhodapkar, 2013), either early in cancer progression or early in the application of immunotherapy. Rather than susceptible versus non-susceptible, we are interested in modeling two ways for how cancer cells evade killer T-cells. The two strategies available to the cancer cells correspond to the two major subsets of immune-resistant cancer cells proposed by Gajewski et al. (2013). The first represents a non-cooperative, selfish strategy where the cancer cell using this strategy enjoys some resistance to the immune system, but in a manner that has no direct influence on other cancer cells. Biologically, this models resistance strategies that involve the cancer cells down-regulating or ceasing to present the antigen required for the Tcell to recognize the cancer cell. This amounts to the cancer cells evolving camouflage and crypticity (Maeurer et al., 1996; Seliger et al., 1997; Hicklin et al., 1999; Johnsen et al., 1999). The second strategy amounts to cooperation as the cancer cells shut down the immune response as a collective public good. Biologically, this models resistance strategies where the cancer cell co-opts signaling pathways by directly signaling the T-cells to cease or by signaling macrophages and/or helper T-cells to cease supporting the production and entraining of killer T-cells. The collective consequence of this second strategy is immunosuppression within the tumor Stewart and Smyth, 2011).

In what follows we develop a predator-prey-like population model that includes the two types of cancer cells and the T-cells. Like Babbs (2012) and Kareva and Berezovskaya (2015), we see the interaction between cancer cells and the T-cells as a modified predator-prey system. A selfish cell enjoys the protection provided by its cooperative counterparts while providing no benefits to other cancer cells. Cooperative cells work together to suppress the immune system. Their rate of survival increases with the ratio of cooperative cells. They gain no protection from the selfish cells. The interplay of selfishness and cooperation represents a public goods game, selfishness being the individually optimal strategy, while cooperation being the socially optimal one. In our model, any given selfish cell is more likely to survive an encounter with an immune cell than 
a cooperative one. Since cooperative cells gain strength in numbers and selfish cells do not, a tumor comprised of cooperative cancer cells may be worse for the patient.

As specific goals, we examine when one or the other cancer strategy will outcompete the other. We then analyze the dynamics and equlibria of tumors comprised of cancer cells using either the selfish or cooperative strategy. Of considerable interest are extinction thresholds where below a critical size the immune system can drive the cancer extinct whereas above these thresholds the cancer will grow to sizes largely unaffected by the immune system. Using comparative statics, we consider the effects of various parameters corresponding to different forms of therapies on the model. Of specific interest are therapies that may unwittingly switch the stable steady state from selfish to cooperative cancer cells thus worsening the patient's prognosis (Smyth et al. 2006). Section 2 introduces the model. In Section 3 we discuss the steady states and the evolutionary stable strategies (ESS) of the model. In Section 4 we discuss the effects of different therapies. Section 5 concludes this paper.

\section{The model}

We imagine a patient with a clinically detectable primary tumor that may or may not be metastatic. We assume that it is a solid tissue cancer, while not specifying the exact kind of cancer. The model considers the interaction between the cancer cells and the immune system within the tumor. For the moment we are imagining a population of killer T-cells. Our focus will be on the dynamics and steady-state population levels of both cancer and immune cells (Section 3). We then consider how various forms of therapy acting through the model's parameters alter tumor growth and prolong the progression free survival of the patient (Section 4).

We use a series of ordinary differential equations to model two cancer cell phenotypes, selfish and cooperative. Let $x_{s}(t)$ and $x_{c}(t)$ denote the population sizes of the selfish and cooperative types at time $t \in[0, \infty)$, respectively. Let $x(t)=x_{s}(t)+x_{c}(t)$ denote the total tumor mass, and $g(t)=x_{c}(t) / x(t)$ be the proportion of cooperative cells. Let $y(t)$ denote the total amount of killer T-cells (referred to as T-cells, for simplicity) in the tumor. We assume that the cells are well mixed, and so the ratio of the cooperative phenotype in any cell's interaction radius is $g(t)$. We refer to the values of $x_{s}(t), x_{c}(t), x(t)$, and $y(t)$ as population counts. The value $g(t)$ is referred to as the composition of the tumor. We henceforth omit the time variable $t$, whenever it does not cause confusion. For a time varying $z(t)$ we use the usual notation $\dot{z}$ to denote its time derivative.

The change in the number of both cancer cell populations and the total population is given 
by:

$$
\begin{gathered}
\dot{x}_{c}=r_{c}\left(1-\frac{\sqrt{x}}{K}\right) x_{c}-g \mu_{c}(g) \sqrt{x} y, \\
\dot{x}_{s}=r_{s}\left(1-\frac{\sqrt{x}}{K}\right) x_{s}-(1-g) \mu_{s}(g) \sqrt{x} y, \\
\dot{x}=\left((1-g) r_{s}+g r_{c}\right)\left(1-\frac{\sqrt{x}}{K}\right) x-\left((1-g) \mu_{s}(g)+g \mu_{c}(g)\right) \sqrt{x} y,
\end{gathered}
$$

with $r_{s}, r_{c}, K \in \mathbb{R}_{+}$, and functions $\mu_{c}, \mu_{s}:[0,1] \rightarrow \mathbb{R}_{+}$.

The non-negative parameters $r_{s}$ and $r_{c}$ denote the growth rates of the selfish and the cooperative phenotypes, respectively. $K^{2}$ is the environment's carrying capacity of tumor cells. We assume that in the absence of immune reaction (or other exogenous factors, e.g. treatment), the population of cancer cells grows until it reaches its carrying capacity, upon which, the population stabilizes.

Population ecology models generally use $K$ to denote carrying capacity. We use $K^{2}$ for notational convenience. Standard logistic growth models are of the form $1-x / K$, which is a concave curve in $x$, peaking at $K / 2$. In our formulation, $1-\sqrt{x} / K$ allows for more tractable analytic results. It behaves similarly to the standard formulation, as it is concave within the interval $0 \leq x \leq K^{2}$, but peaks at $K^{2} / 4$, quarter of the carrying capacity instead of the standard $K / 2$. Our model therefore assumes that the maximum growth of the population is achieved earlier than in standard models. This is motivated by the observation that in the interior of a tumor, cells proliferate at a significantly slower rate than at the exterior (Adam and Maggelakis, 1989; Foryś and Mokwa-Borkowska, 2005), hence the growth rate of the tumor is maximized for a smaller population. In all other ways we follow the typical assumptions of competitive Lotka-Volterra models.

Note that $x$ is the sum of the population of selfish and cooperative phenotypes, and thus the joint reproduction rate $r_{x}(g)$ is the linear combination of the reproduction rates of the phenotypes using their frequencies as weights.

The negative terms on the right-hand sides of (1), (2), and (3) capture the rate at which tumor cells have lethal encounters with T-cells. The encounter rate is given as $\sqrt{x} y$ to keep the analysis tractable. Note that $\sqrt{x}$ is proportional to the perimeter of a 2-dimensional object of area $x$, and in many cases, $\sqrt{x}$ serves as an approximation for the surface area of a 3-dimensional object of volume (or mass) $x$. Therefore, our formulation can be interpreted as all encounters happening on (or in an area proportional to) the surface region of the tumor (Robertson-Tessi et al., 2012).

The function $\mu_{c}$ denotes the rate at which a cooperative cell is killed by a T-cell upon 
encounter, and the function $\mu_{s}$ denotes the rate with which a selfish cell is killed upon encounter. They are given by:

$$
\begin{gathered}
\mu_{c}(g)=\gamma\left(1-g \varepsilon_{c}\right), \\
\mu_{s}(g)=\gamma\left(1-g \varepsilon_{c}\right)\left(1-\varepsilon_{s}\right),
\end{gathered}
$$

with $\varepsilon_{c}, \varepsilon_{s} \in[0,1]$.

The parameter $\gamma$ denotes the lethality of the T-cells, while parameters $\varepsilon_{s}$ and $\varepsilon_{c}$ describe the resistance of the selfish and the cooperative phenotypes, respectively. The value $\mu_{c}(g)$ is the probability that a cooperative cancer cell, encountering an active T-cell, is destroyed, given composition $g$. The value $\mu_{s}(g)$ has the same interpretation for selfish cells.

In (3) the linear combination of the phenotypical death rates $\mu_{x}(g)=(1-g) \mu_{s}(g)+g \mu_{c}(g)=$ $(1-g) \gamma\left(1-g \varepsilon_{c}\right)\left(1-\varepsilon_{s}\right)+g \gamma\left(1-g \varepsilon_{c}\right)$ is determined using the frequencies as weights. Notice that selfish cancer cells always face a lower death rate since they enjoy the protection provided by the cooperative phenotype. The strength of the collective resistance increases with the frequency of the cooperative phenotype, while the strength of selfish resistance (on top of the selfish individuals benefiting from the cooperative resistance) remains constant.

The factor $1 /\left(1-\varepsilon_{s}\right)=\mu_{c} / \mu_{s}$ represents the advantage of selfishness. A value of 1 , coinciding with zero selfish resistance $\varepsilon_{s}=0$, means selfish cells survive an encounter with a T-cell with the same rate as cooperative cells. The higher the $\varepsilon_{s}$ value, the higher the difference between the survival rates, favoring the selfish phenotype.

Let $\omega \in \mathbb{R}^{+}$denote the maximum tumor mass a patient can survive. Once the total cell count of cancer cells reaches $\omega$, the patient dies. We assume $x(0)<\omega$, meaning that the patient is alive at time 0 . Let $T=\inf \{t \geq 0: x(t) \geq \omega\}]^{1}$ denote the patient's survival time.

The change in the population of T-cells is given by:

$$
\dot{y}=r_{i}\left(1-\frac{y}{L+a \sqrt{x}}\right) y-\delta g \sqrt{x} y
$$

with $a, \delta, L \in \mathbb{R}_{+}$. In the absence of tumor cells, the population of T-cells grows logistically with a growth rate (or replenishment rate) $r_{i}$, and a carrying capacity $L$.

As in De Angelis and Mesin (2001) and De Pillis and Radunskaya (2001), an increased tumor mass increases the immune response. This is modeled by a rise in the carrying capacity of Tcells. We assume that this increase is proportional to the encounter rate per T-cell, $\sqrt{x}$. The non-negative parameter $a$ indicates the strength of the increase. Setting $a=0$ means no change in immune response.

\footnotetext{
${ }^{1} \inf \{\emptyset\}=\infty$.
} 
As a deviation from standard predator-prey models we assume that cooperative cancer cells inhibit the growth of the T-cell population. This captures the immunosuppression effect of a tumor, which in our model is induced only by the cooperative phenotype. The effect on the growth rate of the T-cells is proportional to the number of cooperative cancer cells encountered, $g \sqrt{x}$, factored by a constant $\delta$ that measures the effectiveness of immunosuppression. This linear formalization is also in line with De Angelis and Mesin (2001). For a detailed meta-study on the molecular mechanics of tumor-induced immunosuppression the reader is referred to $\mathrm{Wu}$ et al. (2015).

By rearranging (1) and (2) we can derive the evolution of $g$.

$$
\dot{g}=\left(\frac{\dot{x_{c}}}{x}\right)=\frac{\dot{x}_{c} x-\dot{x} x_{c}}{x^{2}}=g(1-g)\left(\left(r_{c}-r_{s}\right)\left(1-\frac{\sqrt{x}}{K}\right)+\left(g \varepsilon_{c} \varepsilon_{s}-\varepsilon_{s}\right) \frac{y}{\sqrt{x}} \gamma\right) .
$$

In (7) it is stated that the frequency of cooperative cells evolves autonomously as a result of differences of birth and death rates of the two phenotypes. The factors $1-\frac{\sqrt{x}}{K}$, and $\gamma \frac{y}{\sqrt{x}}$ express the relative importance of birth and death rates in determining which phenotype proliferates at a higher rate. For instance, in case of a low T-cell population, the difference between $r_{c}$ and $r_{s}$ determines whether the ratio of the cooperative phenotype increases or decreases. With a high T-cell population, the phenotype with the higher resistance prevails.

The system defined by (3), (6), and (7) is equivalent to the one defined by (1), (2), and (6).

\section{Steady-state analysis}

We now evaluate the presence and stability of various steady states. No steady states exist with both cancer cells types (cooperative and selfish) at positive population sizes. The steady state with no cancer cells and no immune cells is always unstable. The steady state with no cancer cells but the presence of immune cells may or may not be stable. Finally, a variety of steady states exist with positive populations of one cancer cell type and immune cells. Their stability properties can be illustrated using zero-isocline diagrams. We start with the condition that determines which cancer cell type outcompetes the other. The sign of the following expression determines whether a given cancer cell type can both invade and resist invasion from the other type:

$$
\frac{r_{c}}{r_{s}}-\frac{1}{1-\varepsilon_{s}}
$$

If the ratio of reproduction rates is larger than the advantage of selfishness, then the tumor (unless eliminated by the T-cells) is cooperative at the ESS, otherwise, it is selfish. Since a purely cooperative tumor is more aggressive than a purely selfish one thanks to the former's 
ability to suppress immune responses, the composition of the tumor, and therefore the sign of the above expression is crucial for the patient's prognosis.

The formal concepts used in this section are as follows.

Definition 3.1. The triple $\left(x^{*}, y^{*}, g^{*}\right)$ is called a steady state of the dynamic system defined by (3), (6), and (7), if $x^{*}>0$, and $\dot{x}=0, \dot{y}=0$, and $\dot{g}=0$ are all satisfied, or if $x^{*}=0$, and $\dot{y}=0$ is satisfied.

The case separation for $x^{*}>0$ and $x^{*}=0$ is necessary due to the fact that for a given $t \leq 0$, if $x(t)=0$, then $g(t)$ is not defined.

A steady state tumor described by $\left(x^{*}, y^{*}, g^{*}\right)$ is called mixed if $g \in(0,1)$, cooperative if $g^{*}=1$, and selfish if $g^{*}=0$. Cooperative and selfish tumors comprise an ESS if they are linearly stable for deviations in $x$ and $y$, and if they resist an invasion of the other phenotype. Since our model precludes mixed steady states, we only discuss stability for homogeneous tumors.

Definition 3.2. Fix $g$. The set of $(x, y)$ pairs for which $\dot{x}=0$ is called the zero-isocline curve of $x$. The set of $(x, y)$ pairs for which $\dot{y}=0$ is called the zero-isocline curve of $y$.

For $t \geq 0$ the triplet $(x(t), y(t), g(t))$ can be thought of as a snapshot of the tumor and the immune system at time $t$, specifying the tumor size, the immune cell count, and the composition of the tumor. As per Definition 3.1, in a steady state, both population counts and the tumor composition are constant.

Clearly, if $\left(x^{*}, y^{*}, g^{*}\right)$ is a steady state, then $\left(x^{*}, y^{*}\right)$ is located along the zero-isocline curves of both $x$ and $y$, given $g^{*}$. The system has three trivial steady states, as listed in the following proposition.

Proposition 3.3. The following triples are all steady states.

1. $x=0, y=L, g$ is undefined.

2. $x=0, y=0, g$ is undefined.

3. $x=K^{2}, y=0, g \in[0,1]$.

Proof. All three cases follow from (3), (6), and (7).

In Proposition 3.3, Case 1 describes a cancer-free patient with T-cells matching the body's carrying capacity. This patient is healthy. Case 2 describes a patient who is cancer-free, but has no T-cells. This may be interpreted as an unrelated immune deficiency. This patient is 
cancer-free but is unprotected against diseases due to a lack of T-cells. Since $\omega<K^{2}$, case 3 describes a patient who has already succumbed to cancer, and has no immune protection.

From (7) one can deduce that both $g=0$ and $g=1$ guarantee the compositional stability of the tumor, meaning that $\dot{g}=0$ is assured. We subdivide this section into three subsections: we briefly show non-existence of steady states in mixed tumors before going on to discuss steady states in selfish and cooperative tumors, respectively.

\subsection{Nonexistence of steady states in mixed tumors}

In this subsection we show that unless the advantage of selfishness happens to be the same as the ratio of reproduction rates, then two steady state conditions, the size of the tumor being constant, and its composition being constant can only be satisfied for purely selfish or purely cooperative tumors.

Proposition 3.4. Suppose that $0<g<1$, and $0<x<K^{2}$.

- If we have $\frac{r_{c}}{r_{s}}>\frac{1}{1-\varepsilon_{s}}$ and $\dot{x}=0$, then it holds that $\dot{g}>0$.

- If we have $\frac{r_{c}}{r_{s}}<\frac{1}{1-\varepsilon_{s}}$ and $\dot{x}=0$, then it holds that $\dot{g}<0$.

Proof. First we consider $r_{c}\left(1-\varepsilon_{s}\right)>r_{s}$. Since $\dot{x}=0$, we must have $\dot{x}_{c}=-\dot{x}_{s}$. If $\dot{x}_{c}>0$ and $\dot{x}_{s}<0$, then $\dot{g}>0$ holds, since the cooperative population is rising and the selfish one dwindling. Suppose that $\dot{x}_{s}>0$. Then we have

$$
0<(1-g) r_{s}\left(1-\frac{\sqrt{x}}{K}\right)-(1-g) \gamma \frac{y}{\sqrt{x}}\left(1-g \varepsilon_{c}\right)\left(1-\varepsilon_{s}\right) .
$$

We multiply by $g /(1-g)$, divide by $\left(1-\varepsilon_{s}\right)$ and invoke $r_{c}\left(1-\varepsilon_{s}\right)>r_{s}$ to get

$$
0<g \frac{r_{s}}{1-\varepsilon_{s}}\left(1-\frac{\sqrt{x}}{K}\right)-g \gamma \frac{y}{\sqrt{x}}\left(1-g \varepsilon_{c}\right)<g r_{c}\left(1-\frac{\sqrt{x}}{K}\right)-g \gamma \frac{y}{\sqrt{x}}\left(1-g \varepsilon_{c}\right)=\dot{x}_{c},
$$

meaning that $\dot{x}_{c}>0$, contradicting $\dot{x}=0$, since both population counts are rising. Therefore, $\dot{x}_{s}<0$ and $\dot{x}_{c}>0$ must hold, implying $\dot{g}>0$.

The case $r_{c}\left(1-\varepsilon_{s}\right)<r_{s}$ follows via similar arguments.

Proposition 3.4 states that constant tumor size cannot coexist with constant tumor composition. Moreover, if the ratio of the reproduction rates $r_{c} / r_{s}$ is higher than the advantage of selfishness, $1 /\left(1-\varepsilon_{s}\right)$, then constant tumor size implies higher relative proliferation of the cooperative cells. If the ratio of reproduction rates is lower, the opposite holds, and the selfish cells will outcompete the cooperative cells. Therefore, no non-trivial steady states exist in which the tumor is a mix of the two phenotypes (the knife-edge case $r_{c} / r_{s}=1 /\left(1-\varepsilon_{s}\right)$ is not considered). 


\subsection{Steady states in selfish tumors}

We now consider selfish tumors. We show that steady states in this case correspond to the solution set of a second-degree polynomial in $\sqrt{x}$. If there are two solutions, the one with a larger tumor population is stable if and only if the tumor population exceeds the growthmaximizing value, a quarter of the carrying capacity. The steady state with the lower tumor cell population is unstable and functions as a strong Allee threshold (Taylor and Hastings, 2005) below which tumor growth is negative.

Proposition 3.5. The triple $\left(x^{*}, y^{*}, 0\right)$ is a steady state, if $x^{*}$ solves the following equation

$$
0=-\frac{r_{s}}{K} x^{*}+\sqrt{x^{*}}\left(r_{s}-a \gamma\left(1-\varepsilon_{s}\right)\right)-\gamma\left(1-\varepsilon_{s}\right) L
$$

and $y^{*}=L+a \sqrt{x^{*}}$.

Proof. Since $g=0$ the zero-isocline of $y$ is simply $y=L+a \sqrt{x}$, the zero-isocline of $x$ is $y=\sqrt{x}\left(1-\frac{\sqrt{x}}{K}\right) \frac{r_{s}}{\gamma\left(1-\varepsilon_{s}\right)}$. Setting the two right hand sides equal and rearranging gives

$$
0=-\frac{r_{s}}{K} x^{*}+\sqrt{x^{*}}\left(r_{s}-a \gamma\left(1-\varepsilon_{s}\right)\right)-\gamma\left(1-\varepsilon_{s}\right) L
$$

Let

$$
D_{s}=\left(r_{s}-a \gamma\left(1-\varepsilon_{s}\right)\right)^{2}-4 \frac{L}{K} r_{s} \gamma\left(1-\varepsilon_{s}\right)
$$

denote the value of the discriminant. Then, the exact values of the steady states are as follows:

- if $D_{s}>0$, there are two steady states, $\left(x_{s 1}^{*}, y_{s 1}^{*}, 0\right)$, and $\left(x_{s 2}^{*}, y_{s 2}^{*}, 0\right)$, with

$$
\sqrt{x_{s 1, s 2}^{*}}=\frac{\left(r_{s}-a \gamma\left(1-\varepsilon_{s}\right)\right) \pm \sqrt{D_{s}}}{2 \frac{r_{s}}{K}}
$$

and $y_{s 1, s 2}^{*}=L+a \sqrt{x_{s 1, s 2}^{*}}$, as long as $x_{s 1, s 2}^{*}>0$.

- if $D_{s}=0$, there is a single steady state, $\left(x_{s 0}^{*}, y_{s 0}^{*}, 0\right)$, where $x_{s 0}^{*}=4 \frac{K L}{r_{s}} \gamma\left(1-\varepsilon_{s}\right)$, and $y_{s 0}^{*}=L+a \sqrt{x_{s 0}^{*}}$, as long as $x_{s 0}^{*}>0$.

- if $D_{s}<0$ (or if $x_{s 1, s 2}^{*}<0$ ), there are no non-trivial steady states in selfish tumors.

The zero-isoclines intersect only if the discriminant condition of Proposition $3.5, D_{s} \geq 0$ is satisfied, with two points of intersection if $D_{s}>0$, and one point of intersection if $D_{s}=0$. 
Proposition 3.6. Let $D_{s}>0$. If $x_{s 1}^{*}>\frac{K^{2}}{4}$, then $\left(x_{s 1}^{*}, y_{s 1}^{*}, 0\right)$ is stable in $x$ and $y$.

The proof is shown as an appendix. It relies on showing that the elements of the problem's Jacobian matrix take the following signs:

$$
J\left(x_{s 1}^{*}, y_{s 1}^{*}\right)=\left(\begin{array}{cc}
- & - \\
+ & -
\end{array}\right)
$$

with the negativity of the top left element hinging on the condition $x_{s 1}^{*}>K^{2} / 4$. Thus, the trace of the Jacobian is negative, and the determinant positive, meaning that the steady state is stable. As long as the advantage of selfishness is larger than the ratio of phenotypical reproduction rates, this is an ESS.

Corollary 3.7. Let $D_{s}>0$. If $x_{s 1}^{*}>K^{2} / 4$, and if $r_{c} / r_{s}<1 /\left(1-\varepsilon_{s}\right)$ then $\left(x_{s 1}^{*}, y_{s 1}^{*}, 0\right)$ is an ESS.

Proof. By Proposition 3.6 we have linear stability in $x$ and $y$, and since the advantage of selfishness is higher than the ratio of reproduction rates, Proposition 3.4 ensures that the selfish phenotype resists invasion by the cooperative one.

We illustrate the steady states in the selfish case, as well as some typical dynamic properties in the following example.

Example 3.8. Consider a tumor of selfish cells and the following parameter values: $K=25$, $L=20, r_{s}=0.2, \gamma=0.1, \varepsilon_{s}=0.7$. The interpretation is the following. The carrying capacity of tumor cells is $25^{2}=625$, significantly higher than that of T-cells, at 20 . The unconstrained rate of proliferation is $20 \%$. In $10 \%$ of encounters, T-cells become activated, and the cancer cells resist destruction with a rate of $70 \%$ once a T-cell is activated, for an effective kill rate of $3 \%$ per encounter. These parameters satisfy $D_{s}>0$, hence we have a total of five steady states. Fix $a=1$ and $\omega=470$.

Figure 1 shows the zero-isoclines, phase diagram, and steady states of this system. Black dots denote the trivial steady states of Proposition 3.3 , $(0,0),(0, L),\left(K^{2}, 0\right)$, and red ones denote the steady states where both the tumor size and the T-cell count is non-zero from Proposition 3.5 . In figure 2 we plot the growth of the tumor population as a function of population size, moving along the $y$ zero-isocline, a smooth function of $x$. Notice that the tumor's growth rate along the zero-isocline is maximized near a cancer cell population size of 150 , or $24 \%$ of carrying capacity. The change of population, compared to distances between steady states, is small, a useful property for discretization of the model in conducting simulations. By Proposition 3.5, 


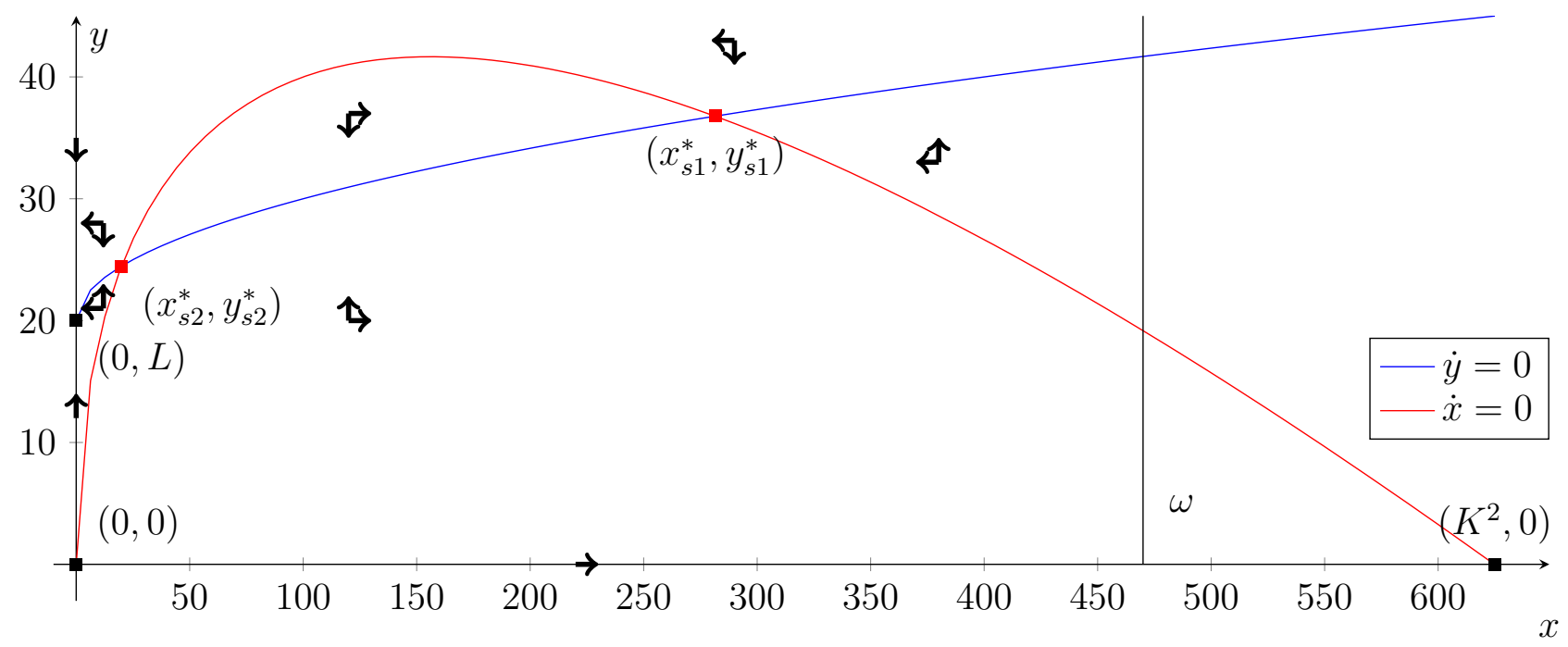

Figure 1: Phase diagram of a selfish tumor with a positive discriminant. The steady state with a lower cancer cell population is unstable, and serves as an Allee threshold. The steady state with a higher cancer cell population is stable and is below the lethal tumor mass. Patient could survive with the cancer burden indefinitely.

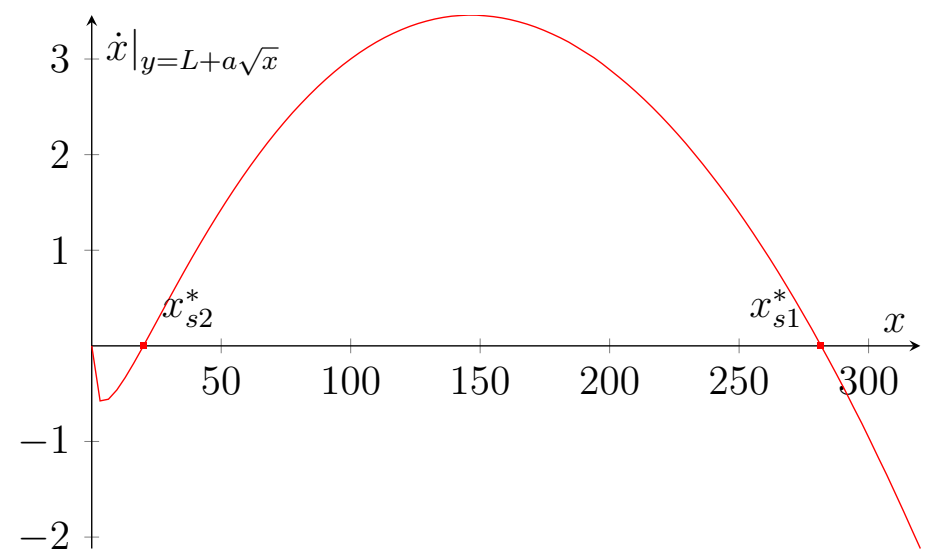

Figure 2: The growth rate of a selfish tumor along the $y$ zero-isocline. $x_{s 2}^{*}$ serves as a strong Allee threshold. Tumor population stabilizes at $x_{s 1}^{*}$. Tumor growth is maximized at the quarter of the tumor's carrying capacity.

we have the two steady states, $x_{s 1}^{*}=281.6, y_{s 1}^{*}=36.78$ and $x_{s 2}^{*}=20, y_{s 2}^{*}=24.47$. The interpretation is the following. At the theoretical maximum population count, the cancer cells are at $45 \%$ carrying capacity, while the T-cells are at $184 \%$ of $L$, meaning that the immune reaction to the tumor burden is significant.

At the Allee threshold, the tumor burden is $3.2 \%$ of carrying capacity, whereas T-cells are at $122 \%$ of $L$.

Below a tumor burden of 20, a strong immune system overcomes and eliminates the cancer despite its relatively large growth potential. Above the Allee threshold, the reproduction of cancer cells offsets even a strong immune response. Tumor growth is maximized for values 
of about 150, and stops at the theoretical maximum of 281.6. The model has two reasons for this absence of growth. First, a larger tumor induces a larger immune reaction that directly hinders the proliferation of cancer cells, and second, a larger tumor will face a lower abundance of resources that the cells use to reproduce.

Note that if for any reason (i.e. hidden tumors, or immune deficiency) a smaller tumor burden can manage to exceed the Allee threshold, reversing tumor growth becomes harder and harder as time passes. Another implication of this example is that if a successful treatment eradicates a large fraction of the tumor, but not enough to go below the Allee threshold, then in time the cancer will return.

Example 3.9. Consider the same parameter values as example 3.8 , but with $\gamma=0.2$ instead of 0.1 , corresponding to a stronger immune system. Then $D_{s}<0$, and the zero-isocline of $y$ lies above the zero-isocline of $x$. Figure 3 shows the appropriate phase diagram. As per Proposition 3.5, there are no non-trivial steady states. Note that the steady state, $(0, L)$ behaves as an attractor for every initial condition with $y(0)>0$, meaning that the cancer is always eradicated (unless the lethal tumor mass is reached before that happens).

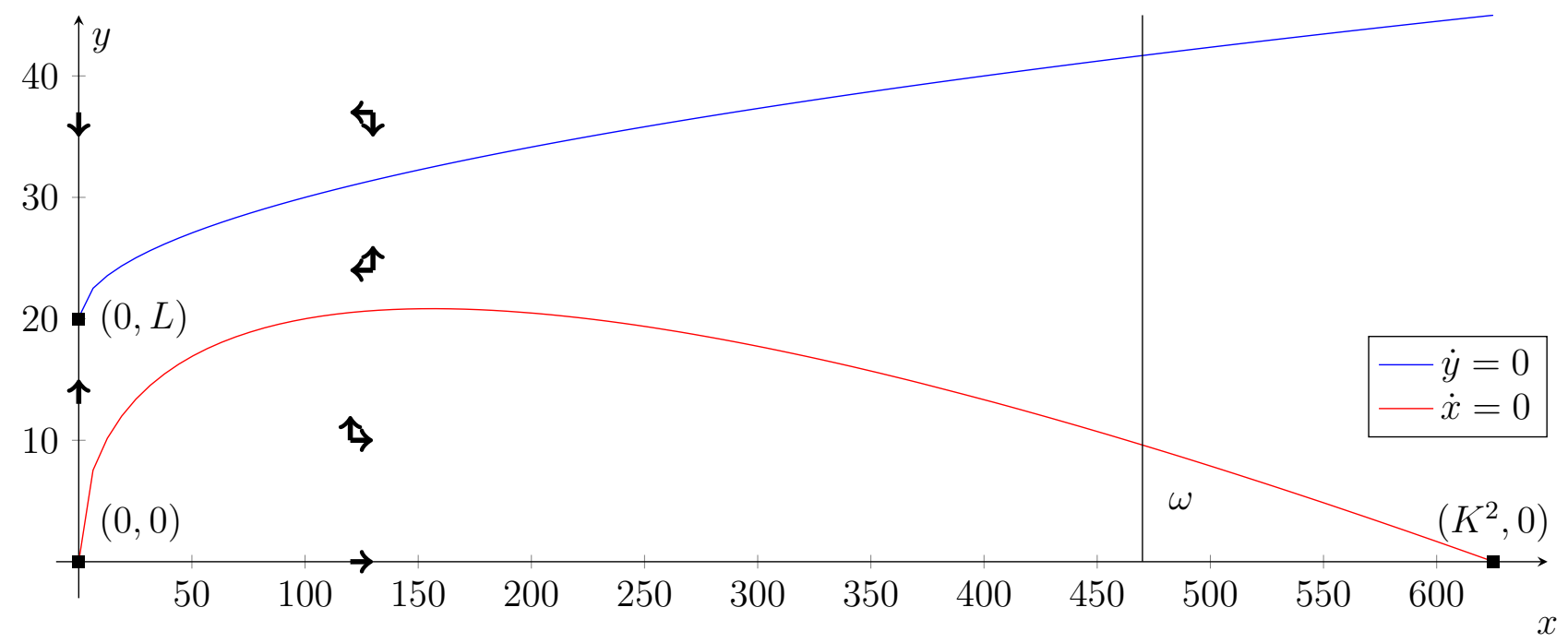

Figure 3: Phase diagram of a selfish tumor with a negative discriminant. The zero-isoclines do not intersect. A healthy immune system drives the cancer cells to extinction. Patient is expected to become cancer free.

\subsection{Cooperative steady states}

We now examine tumors composed of cooperative cancer cells. As before, the set of steady states corresponds to the solution set of a second degree polynomial in $\sqrt{x}$, and in case of two 
cooperative steady states the one with a higher cancer cell population count is stable if the number of cancer cells exceed the growth maximizing value of $K^{2} / 4$.

Proposition 3.10. The triple $\left(x^{*}, y^{*}, 1\right)$ is a steady state, if $x^{*}$ solves the following equation:

$$
0=\left(-\frac{r_{c}}{K}+a \gamma\left(1-\varepsilon_{c}\right) \frac{\delta}{r_{i}}\right) x^{*}+\left(r_{c}-\gamma\left(1-\varepsilon_{c}\right)\left(a-\frac{\delta}{r_{i}} L\right)\right) \sqrt{x^{*}}-\gamma\left(1-\varepsilon_{c}\right) L,
$$

and $y^{*}=\sqrt{x^{*}}\left(1-\frac{\sqrt{x^{*}}}{K}\right) \frac{r_{c}}{\gamma\left(1-\varepsilon_{c}\right)}$.

Proof. Similarly to the selfish case, we calculate the zero-isocline curves by setting (3) and (6) to 0 and substituting $g=1$ :

$$
\dot{y}=0 \Leftrightarrow y=(L+a \sqrt{x})\left(1-\frac{\delta}{r_{i}} \sqrt{x}\right)=L+a \sqrt{x}\left(a-\frac{\delta L}{r_{i}}\right)-a \frac{\delta}{r_{i}} x,
$$

and

$$
\dot{x}=0 \Leftrightarrow y=\sqrt{x}\left(1-\frac{\sqrt{x}}{K}\right) \frac{r_{c}}{\gamma\left(1-\varepsilon_{c}\right)} .
$$

Setting the right hand sides equal and rearranging gives

$$
\frac{\dot{x}}{\sqrt{x}}=\left(-\frac{r_{c}}{K}+a \gamma\left(1-\varepsilon_{c}\right) \frac{\delta}{r_{i}}\right) x+\left(r_{c}-\gamma\left(1-\varepsilon_{c}\right)\left(a-\frac{\delta}{r_{i}} L\right)\right) \sqrt{x}-\gamma\left(1-\varepsilon_{c}\right) L,
$$

hence the roots of the above expression are the steady-state tumor masses.

Let

$$
D_{c}=\left(r_{c}-\gamma\left(1-\varepsilon_{c}\right)\left(a-\frac{\delta}{r_{i}} L\right)\right)^{2}-4 \frac{L}{K} r_{c} \gamma\left(1-\varepsilon_{c}\right)+4 a \gamma^{2}\left(1-\varepsilon_{c}\right)^{2} \frac{\delta}{r_{i}} L
$$

denote the value of the discriminant in the cooperative case. The exact values of the steady states are as follows:

- if $D_{c}>0$, there are two steady states, $\left(x_{c 1}^{*}, y_{c 2}^{*}, 1\right)$, and $\left(x_{c 2}^{*}, y_{c 2}^{*}, 1\right)$, where

$$
\sqrt{x_{c 1, c 2}^{*}}=\frac{\left(r_{c}-\gamma\left(1-\varepsilon_{c}\right)\left(a-\frac{\delta}{r_{i}} L\right)\right) \pm \sqrt{D_{c}}}{2 \frac{r_{c}}{K}-2 \gamma\left(1-\varepsilon_{c}\right) \frac{\delta}{r_{i}}},
$$

and $y_{c 1, c 2}^{*}=\left(L+a \sqrt{x_{c 1, c 2}}\right)\left(1-\frac{\delta}{r_{i}} \sqrt{x^{*}}{ }_{c 1, c 2}\right)$, provided that $y_{c 1}^{*}$ and $y_{c 2}^{*}$ are positive.

- if $D_{c}=0$, there is a single steady state, $\left(x_{c 0}^{*}, y_{c 0}^{*}, 1\right)$, where $\sqrt{x_{c 0}^{*}}=\frac{\left(r_{c}-\gamma\left(1-\varepsilon_{c}\right)\left(a-\frac{\delta}{r_{i}} L\right)\right)}{2 \frac{r_{c}}{K}-2 \gamma\left(1-\varepsilon_{c}\right) \frac{\delta}{r_{i}}}$, and $y_{c 0}^{*}=\left(L+a \sqrt{x_{c 0}^{*}}\right)\left(1-\frac{\delta}{r_{i}} \sqrt{x_{c 0}^{*}}\right)$, provided that $y_{c 0}^{*}$ is positive.

- if $D_{c}<0$, there are no steady states. 
By analyzing the zero-isoclines in both cases one can see that the main difference between the purely selfish and the purely cooperative case comes from the suppression of T-cells by the cooperative phenotype. Notice that the T-cell zero-isocline includes a factor of $1-\left(\delta / r_{i}\right) \sqrt{x}$. This factor is increasing in T-cell reproduction rate $r_{i}$ and decreasing in the suppression parameter $\delta$, as well as the encounter rate per T-cell $\sqrt{x}$. Hence a positive $\delta$ leads to a diminished T-cell population as a direct result of suppression.

Note however, that immunosuppression also indirectly raises the T-cell population. A lower T-cell population permits a higher steady-state tumor size, which in turn raises the T-cell population via an increased carrying capacity of T-cells. This induces a higher effective replenishment rate of the T-cell population.

We now state the conditions of a cooperative stable steady state.

Proposition 3.11. Let $D_{c}>0$. If $x_{c 1}^{*}>K^{2} / 4$, then $\left(x_{c 1}^{*}, y_{c 1}^{*}, 1\right)$ is linearly stable in $x$ and $y$.

The proof is shown as an appendix. The negativity of the Jacobian's trace is assured by the $x_{c 1}^{*}>K^{2} / 4$ condition, whereas the positivity of the determinant is due to the fact that the T-cell zero-isocline intersects the cancer's zero-isocline from below. If the advantage of selfishness is lower than the ratio of phenotypical reproduction rates, this steady state is also an ESS.

Corollary 3.12. Let $D_{c}>0$. If $x_{s 1}^{*}>K^{2} / 4$, and if $r_{c} / r_{s}>1 /\left(1-\varepsilon_{s}\right)$ then $\left(x_{c 1}^{*}, y_{c 1}^{*}, 1\right)$ is an ESS.

Proof. By Proposition 3.11 we have linear stability in $x$ and $y$, and since the advantage of selfishness is lower than the ratio of reproduction rates, Proposition 3.4 ensures that the cooperative phenotype resists an invasion by the selfish phenotype.

Together, Corollaries 3.7 and 3.12 mean that there is always a unique ESS of the system, and the phenotype therein is determined by the advantage of selfishness.

Example 3.13. Consider a tumor with only cooperative cells and the following parameter values: $K=25, L=20, r_{c}=0.2, r_{i}=0.1, \gamma=0.1, \varepsilon_{c}=0.7, a=1$. We retain the values for the reproduction rate and resistance parameter of example 3.8 so that we can better demonstrate the effect of immunosuppression. Figure 4 shows the isocline diagram, plotted for $\delta=0.003$. The interpretation is that T-cells are killed with a rate of $0.3 \%$ per encounter. In this example, the cancer cell population and the T-cell population act as competitors, as both zero-isoclines have negative slopes at the stable non-trivial steady-state. Notice that the cancer's zero-isocline is unaffected by the change in the suppression parameter and remains a concave curve, as in 


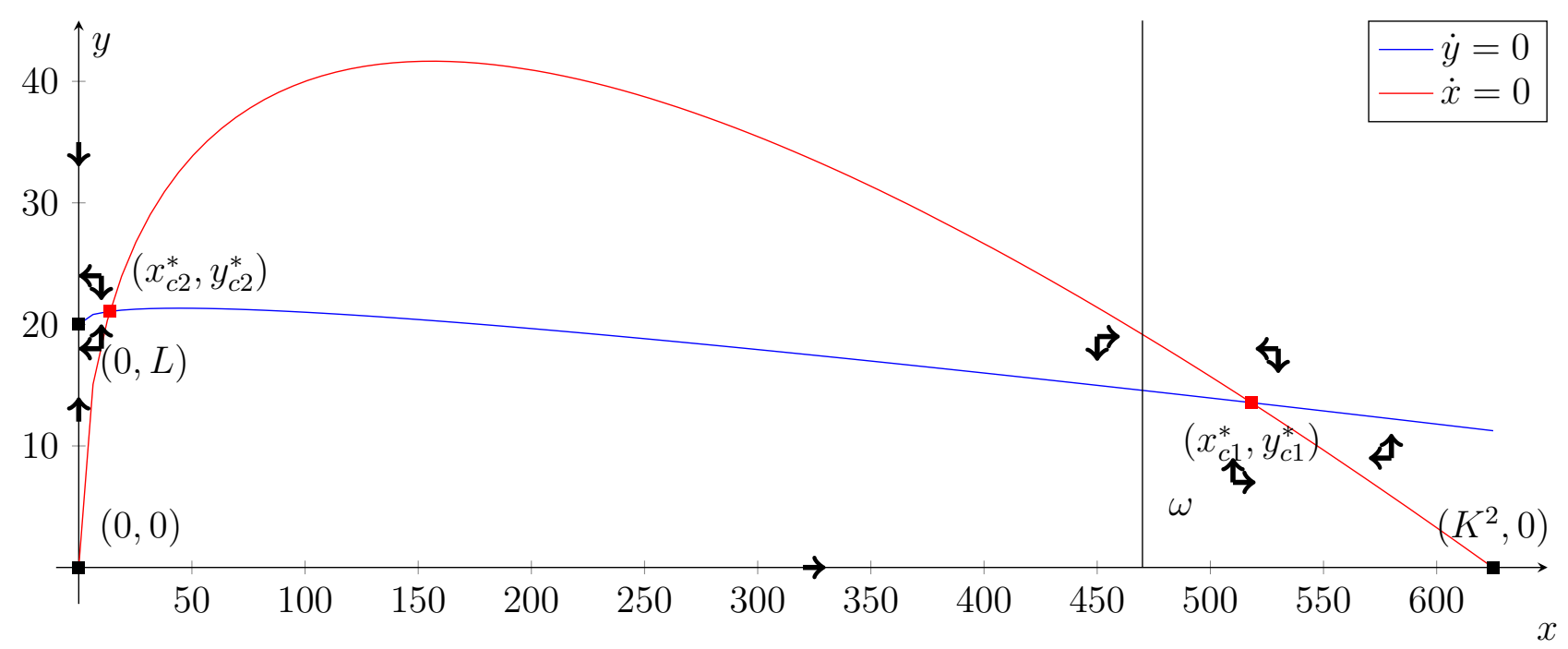

Figure 4: Phase diagram of a cooperative tumor with two non-trivial steady states. The steady state with lower cancer cell population is unstable, and serves as an Allee threshold. The steady state with higher cancer cell population mass is stable, and is above the lethal tumor mass. Patient prognosis is poor.

example 3.8. However, with $\delta>0$, the zero-isocline for $y$ has an upward sloping part and becomes downward sloping. Two non-trivial steady states exist.

In figure 5 we plot the same diagram with $\delta=0.005$, corresponding to a stronger immunosuppression ability of the cooperative phenotype (for this calibration, the zero-isocline of $y$ reduces to a line). In this case, the steady state $\left(x_{c 1}^{*}, y_{c 1}^{*}\right)$ is out of bounds, since $x_{c 1}^{*}>K^{2}$ and $y_{c 1}^{*}<0$, meaning that we only get one non-trivial steady state, $\left(x_{c 2}^{*}, y_{c 2}^{*}\right)$, a saddle point, leaving $(0, L)$ and $\left(K^{2}, 0\right)$ as stable steady states. A complete suppression of the immune system is therefore possible.

\section{Treatment effects}

The principal objectives behind cancer modeling are to improve and inform the research into therapy. Here we explore the implications of our model for cancer therapy strategies. We do this by investigating how a change in the tumor's micro-environment affects the tumor and the immune system and then draw conclusions on their influence on the patient's prognosis.

Patient outcome in our model can be measured as survival time and/or as the tumor's steadystate size. Our survival time measure relates to what is known as progression free survival time following the initiation of therapy. This can be actual time before patient death, or it may represent the time before another line of therapy becomes necessary. The steady-state size of the primary tumor may be of interest as this may correlate with the likelihood or rate of 


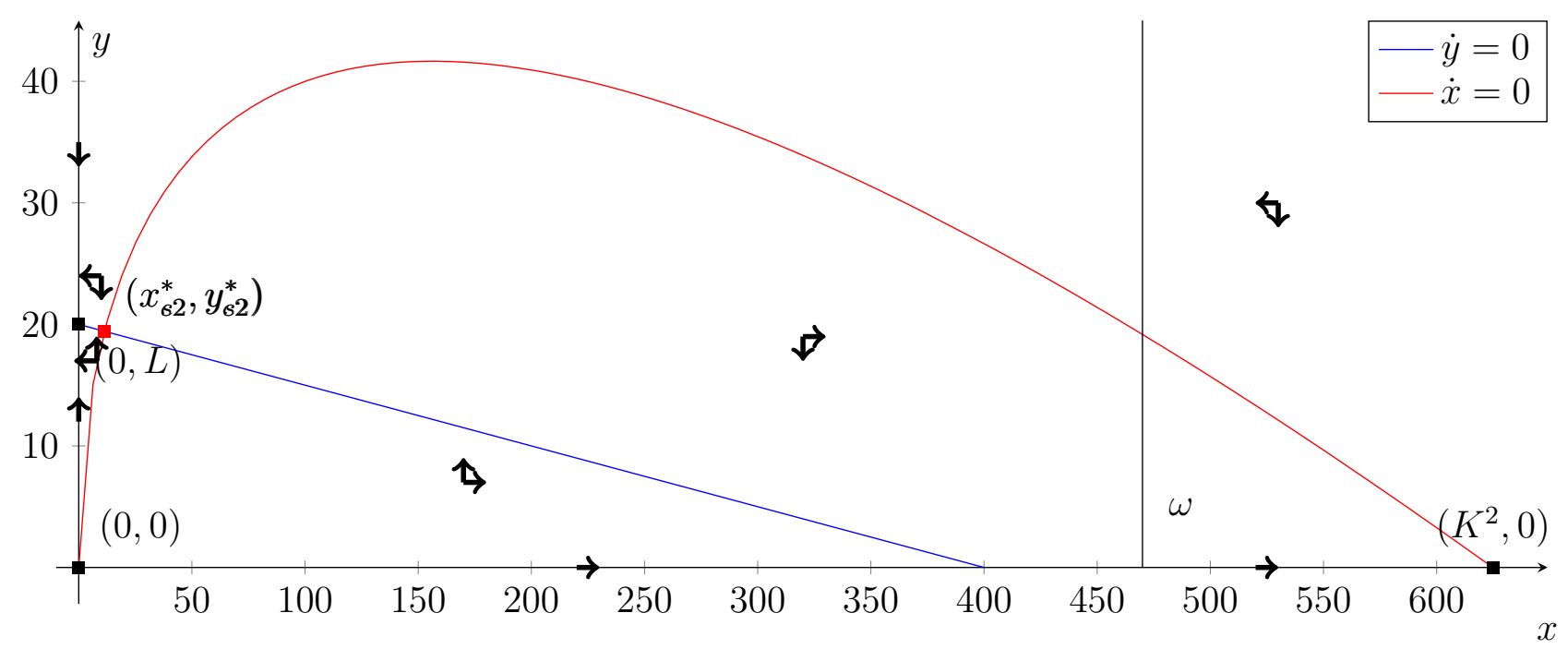

Figure 5: Phase diagram of a cooperative tumor with a single non-trivial steady state. The zero-isoclines intersect in the positive quadrant only once. A complete suppression of the immune system becomes possible. Patient prognosis is grave.

metastases. We can use our model to examine the nature and effectiveness of various forms of immunotherapy. Specifically, we are interested in how changes in model parameter values and outcomes can be interpreted from a therapeutic standpoint. Immunotherapy likely influences three key parameters of the model:

1. Increasing $\gamma$, the rate at which cancer cells have lethal encounters with T-cells. In immunotherapy this is often accomplished by using retroviruses or vaccines that tag the cancer cells with an antigen that can be detected by the patient's immune system, thus eliciting the production of antigen specific T-cells. Alternatively, immune cells from the patient may be entrained and cultured in vitro to recognize cancer cells collected through biopsy. These immune cells are then injected into the patient.

2. Decreasing $\delta$, the suppression parameter of the cooperative phenotype. Targeted therapy can be used to lower the cooperative phenotype's ability to suppress the immune system. Such therapy by neutralizing the cancer cells' signaling or by killing cells that overexpress PDL-1, either reduce $\delta$, or strongly select against cancer cells with high values of $\delta$.

3. Decreasing $\varepsilon_{c}$ and $\varepsilon_{s}$, the resistance rates of the two phenotypes. The T-cells kill cancer cells through direct contact, and the injection of proteins that initiate apoptosis (cell death). Cancer cells may exaggerate glycolysis to produce lactic acid. The secreted lactic acid by reducing $\mathrm{Ph}$ creates a protective moat that impairs contact with an encountered T-cell. This is often associated with the upregulation of carboanhydrous IX (CAIX) that assists the cancer cell in maintaining and surviving an acidic environment. Buffer therapies can be added to the 
immunotherapy as a means of raising the $\mathrm{Ph}$ of the tumor and rendering the cancer cells' acidic moat ineffective to lower $\varepsilon_{c}$ (Ibrahim-Hashim et al., 2017). Checkpoint inhibitors may unmask the selfish phenotype, making the selfish phenotype more susceptible to destruction by the T-cells (Pardoll, 2012).

With such therapies in mind, in this section we consider the effects of these key parameters on the trajectory of tumor growth and the steady-state size of the tumor. We do this by using comparative statics and simulations on a discrete version of our model. Our conclusions are as follows.

1. Increased immune system efficiency and/or decreased cooperative resistance prolongs the patient's survival time and lowers tumor mass at the stable steady state.

2. Decreased immunosuppression prolongs the patient's survival time in tumors that feature the cooperative phenotype. It decreases tumor mass at the stable steady state, if it is cooperative, and has no effect on the stable steady state, if it is selfish.

3. Decreased crypticity has ambiguous effects. It may increase or decrease the patient's survival time and increase or decrease tumor mass at the stable steady state.

The first two findings are in line with expectations, while the third may seem counterintuitive. The reason for this is that reduced crypticity lowers the advantage of selfishness, and thus pushes the cancer cells towards an ESS with cooperative cancer cells. If the tumor becomes cooperative instead of selfish at the stable steady state, the patient's prognosis may be worse due to the cooperative phenotype's immunosuppression. Treatments that increase the immune system's efficiency, decrease the cooperative resistance, or decrease immunosuppression have no effect on the advantage of selfishness. Hence, these treatment strategies do not influence the tumor's steady-state composition and there is no ambiguity in their benefits on the patient's prognosis.

The remainder of this section formalizes, extends, and illustrates the above results.

\subsection{Effective treatment strategies}

Any treatment that does not change the advantage of selfishness can be shown to be effective. We first formalize the effects of the parameters $\gamma$ and $\varepsilon_{c}$ on the steady-state tumor size.

Proposition 4.1. $\quad$ 1. If $D_{c}$ is positive then, for every parametrization, the following comparative statics hold. 

a. $\frac{\partial x_{c 1}^{*}}{\partial \gamma}<0, \frac{\partial x_{c 2}^{*}}{\partial \gamma}>0$
b. $\frac{\partial x_{c 1}^{*}}{\partial \varepsilon_{c}}>0, \frac{\partial x_{c 2}^{*}}{\partial \varepsilon_{c}}<0$.

2. If $D_{s}$ is positive then, for every parametrization, the following comparative statics hold.
a. $\frac{\partial x_{s 1}^{*}}{\partial \gamma}<0, \frac{\partial x_{s 2}^{*}}{\partial \gamma}>0$,
b. $\frac{\partial x_{s 1}^{*}}{\partial \varepsilon_{c}}=0, \frac{\partial x_{s 2}^{*}}{\partial \varepsilon_{c}}=0$.

Proof. 1.a. By raising $\gamma$, the cancer's zero-isocline is compressed towards the $x$-axis, while the Tcells' zero-isocline stays the same. It follows that the points of intersection move closer together, hence tumor mass decreases at the stable steady state, and the Allee threshold is achieved at a higher tumor mass.

b. Decreasing $\varepsilon_{c}$ has the same effect as increasing $\gamma$.

2.a. Same as 1.a.

b. Changing $\varepsilon_{c}$ has no effect on the zero-isoclines in the selfish case, hence the selfish steady states do not change.

Proposition 4.1 confirms the first point outlined at the beginning of this section. Treating for immune system efficiency and/or cooperative resistance decreases tumor mass at the stable steady state, making metastases less likely. In addition, this treatment increases the Allee threshold, thereby making the cancer population more vulnerable to other forms of treatment (Tobin) et al. 2011). Figure 6 shows the qualitative effects of an increased immune efficiency parameter on the two cooperative steady states with original parameters taken from Example 3.13. The dashed red line shows the original cancer zero-isocline, the solid red line shows the zero-isocline after the increase. Note that in this example the treatment results in the cancer cell population at the stable steady state being lower than the critical tumor mass, hence a patient may survive indefinitely with the tumor burden due to this therapy.

Next, we formulate the relationship between the suppression parameter and steady-state tumor sizes for cooperative tumors.

Proposition 4.2. If $D_{c}$ is positive then, for every parametrization, the following comparative statics hold: $\frac{\partial x_{c 1}^{*}}{\partial \delta}>0, \frac{\partial x_{c 2}^{*}}{\partial \delta}<0$.

Proof. Raising $\delta$ has no effect on the cancer zero-isocline and decreases the slope of the T-cell isocline, hence the points of intersection spread farther apart, meaning that tumor size increases at the stable steady state and decreases at the Allee threshold. 


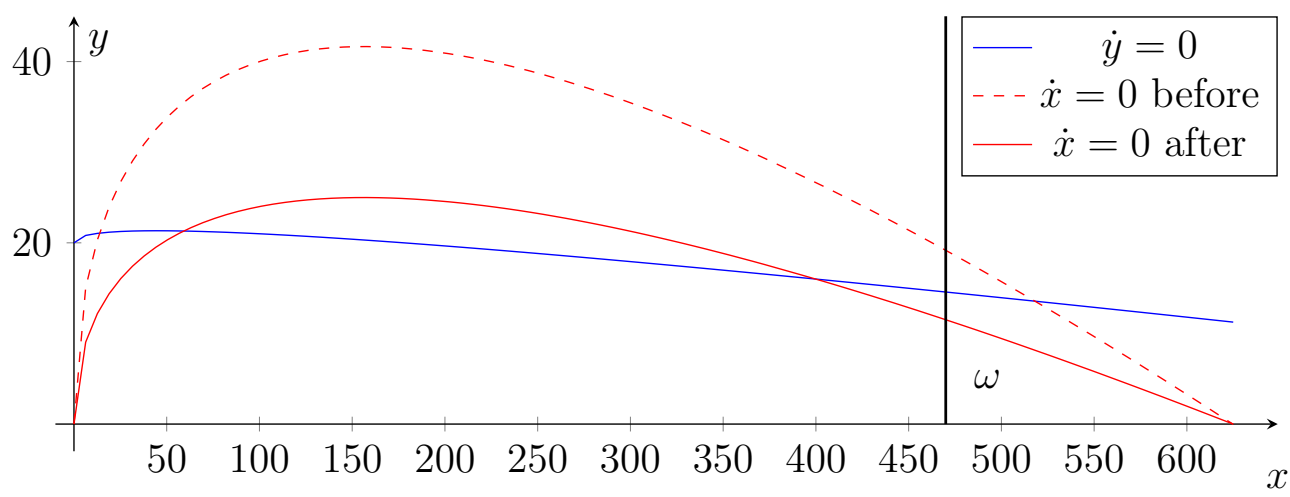

Figure 6: The effect of an increased $\gamma$ on cooperative steady states. The cancer zero-isocline is compressed towards the horizontal axis. Tumor mass becomes higher at the Allee threshold, and lower at the stable steady state. Steady-state tumor mass falls below the lethal value. Patient prognosis improves as a result of the treatment.

Proposition 4.2 confirms the second point highlighted in the beginning of this section. It implies that lowering the immunosuppression parameter via treatment has the same qualitative effect on steady states in cooperative tumors as strengthening the immune system. Clearly, such a treatment has no effect on a purely selfish tumor as the selfish phenotype does not display immunosuppression. Figure 7 shows the qualitative effects of a decreased immunosuppression parameter on the two cooperative steady states with original parameters retained from Example 3.13. The dashed blue line shows the original T-cell zero-isocline, the solid red line shows the zero-isocline after the treatment. Once again, this example shows a treatment that results in the cancer cell population at the stable steady state being lower than the critical tumor mass. We

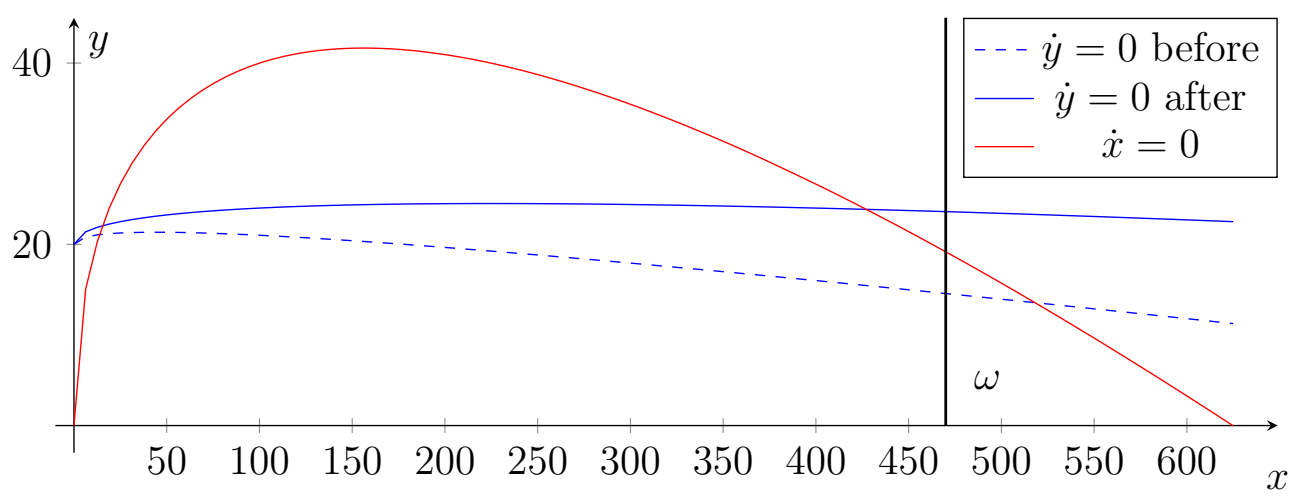

Figure 7: The effect of an decreased $\delta$ on cooperative steady states. The T-cell zero-isocline tilts upward. Tumor mass becomes higher at the Allee threshold, and lower at the stable steady state. Steady-state tumor mass falls below the lethal value. Patient prognosis improves as the result of the treatment.

now consider the effects of therapy on the survival time of the patient, $T$, a variable determining the viability of a treatment strategy. We develop a round of simulations in the discretized version of the model to obtain approximations of the effects of treatments via $\gamma$ and in $\delta$ on survival 
time.

The configurations used are as follows: $K=25, L=20, r_{s}=0.19, r_{c}=0.2, \varepsilon_{s}=0.7, \varepsilon_{c}=1$, $a=1$. We considered three possible critical tumor masses $\omega_{1}=150, \omega_{2}=200, \omega_{3}=250$. The initial conditions of the system are characterized by $x(0)=40, y(0)=20, g(0)=0.7$. Note that recalibrating the model with different parameters and initial conditions does not change the qualitative effects but may lead to an early extinction of the tumor, making survival times infinite. Table 1 contains the corresponding $T_{1}, T_{2}$, and $T_{3}$ values for the different combinations of $\gamma$ and $\delta$. The trends in each table are clear, for all three critical tumor masses, increasing $\gamma$ or

\begin{tabular}{|c|c|c|c|c|c|c|c|c|}
\hline & $T_{1}$ & 0.025 & 0.05 & $\gamma$ & 0.1 & \multicolumn{2}{|c|}{$T_{2}$} & 0.025 \\
\hline \multirow{12}{*}{$\delta$} & 0.001 & 13 & 15 & 20 & 41 & & 0.001 & 17 \\
\hline & 0.003 & 13 & 15 & 19 & 31 & & 0.003 & 16 \\
\hline & 0.005 & 13 & 15 & 18 & 26 & $\delta$ & 0.005 & 16 \\
\hline & 0.007 & 13 & 15 & 17 & 23 & & 0.007 & 16 \\
\hline & 0.009 & 13 & 14 & 17 & 21 & & 0.009 & 16 \\
\hline & & & \multirow{2}{*}{\multicolumn{2}{|c|}{$T_{3}$}} & \multicolumn{4}{|c|}{$\gamma$} \\
\hline & & & & & 0.025 & 0.05 & 0.075 & 0.1 \\
\hline & & & & 0.001 & 20 & 24 & 36 & 90 \\
\hline & & & & 0.003 & 20 & 23 & 31 & 76 \\
\hline & & & $\delta$ & 0.005 & 20 & 23 & 28 & 54 \\
\hline & & & & 0.007 & 19 & 22 & 26 & 39 \\
\hline & & & & 0.009 & 19 & 22 & 25 & 33 \\
\hline
\end{tabular}

Table 1: Survival times for different combinations of $\gamma$ and $\delta$. Patient prognosis improves with increase in $\gamma$ and decrease in $\delta$.

decreasing $\delta$ weakly increases survival time. Clearly, patients live the longest when the immune system is strongest and immunosuppression is weakest, corresponding to the upper right corners of each table. Conversely, when the immune system is weak and immunosuppression is the strong, life expectancy is lowest, corresponding to the bottom left corners of the tables. Furthermore, notice that a stronger immune system means a stronger effect of immunosuppression. All of these observations predicted by our model fall in line with basic intuitions about immunotherapy.

\subsection{Ambiguous effects of treating against cancer crypticity}

Treating for the selfish resistance rate - the crypticity parameter - may have ambiguous effects on the patient. Specifically, we first show that reducing the resistance rate may both increase or 
decrease the tumor population at the stable steady state. On first glance this may seem counterintuitive as it is easy to show that selfish steady states react the same way to a treatment of $\varepsilon_{s}$ as cooperative ones to that of $\varepsilon_{c}$.

Proposition 4.3. If $D_{s}$ is positive then, for every parametrization, the following comparative statics hold: $\partial x_{c 1}^{*} / \partial \varepsilon_{s}>0, \partial x_{c 2}^{*} / \partial \varepsilon_{s}<0$.

Proof. Very similar to Proposition 4.1 1.b.

Proposition 4.3 indicates that marginal changes of the selfish resistance parameter also constitute an effective treatment, as they have no effect on steady-state composition. However, if due to a major change of $\varepsilon_{s}$, the advantage of selfishness becomes lower than the ratio of the reproduction rates, $r_{c} / r_{s}$, then the system's ESS may become cooperative instead of selfish. This may worsen the patient's prognosis. We provide an example to illustrate this.

Example 4.4. Consider the following calibration: $K=25, L=20, r_{c}=0.2, r_{s}=0.19$, $\varepsilon_{c}=\varepsilon_{s}=0.7, \delta=0.001, \gamma=0.1$. In this case, $r_{c}\left(1-\varepsilon_{s}\right)=0.06<0.19=r_{s}$, so the advantage of selfishness is substantial. The system's only ESS is $\left(x_{s 1}^{*}, y_{s 1}^{*}, 0\right)$ where the tumor size is $x_{s 1}^{*}=261.5$. At the steady state, the cancer cells are at $42 \%$ of carrying capacity, while the T-cell population is $36.17,181 \%$ of carrying capacity.

Suppose that the selfish phenotype's defenses are fully eliminated, i.e. $\varepsilon_{s}^{\prime}=0$ due to a successful treatment. The interpretation is that cancer cells are no longer able to block immune checkpoints. As a result, the advantage of selfishness is minimized, and $r_{c}\left(1-\varepsilon_{s}^{\prime}\right)>r_{s}$. The new ESS is $\left(x_{c 1}^{*}, y_{c 1}^{*}, 1\right)$. The tumor size is $x_{c 1}^{*}=349.2$, or $56 \%$ carrying capacity, which is a $34 \%$ increase in size. For the number of T-cells, we have 31.45, 157\% of carrying capacity, a decrease of $13 \%$ in population. Additionally, we face a decrease in the Allee threshold, from $x_{s 2}^{*}=23.8$, or $3.8 \%$ carrying capacity to $x_{c 2}^{*}=17.4,2.8 \%$ carrying capacity, a decrease of $27 \%$. The tumor has switched from selfish to cooperative cancer cells.

Example 4.4 shows the importance of treatment effects on tumor composition: a treatment of the selfish phenotype's crypticity has led to an increase in the steady-state tumor size. However, this is not a general rule. As per Proposition 4.3, such a treatment always decreases tumor size if the tumor composition at the steady state is preserved.

The effects of treatment on the patient's prognosis are also ambiguous. Consider once again the configuration used for the previous simulation: $K=25, L=20, r_{s}=0.19, r_{c}=0.2, \varepsilon_{c}=1$, $a=1$, complete with $\gamma=0.1$ and $\delta=0.005$, and critical tumor masses $\omega_{1}=150, \omega_{2}=200$, $\omega_{3}=250$. The initial conditions of the system are $x(0)=40, y(0)=20, g(0)=0.7$.the above 
parametrization with $\gamma=0.1$ and $\delta=0.005$. Table 2 contains the $T_{1}, T_{2}$, and $T_{3}$ levels for different values of $\varepsilon_{s}$ with the same initial conditions as before. Unsurprisingly, survival times

\begin{tabular}{cccc}
\hline$\varepsilon_{s}$ & $T_{1}$ & $T_{2}$ & $T_{3}$ \\
\hline 0 & 23 & 29 & 33 \\
0.1 & 24 & 30 & 35 \\
0.2 & 25 & 31 & 37 \\
0.3 & 26 & 33 & 41 \\
0.4 & 27 & 36 & $\infty$ \\
0.5 & 27 & 45 & $\infty$ \\
0.6 & 28 & $\infty$ & $\infty$ \\
0.7 & 26 & 37 & 54 \\
0.8 & 24 & 32 & 39 \\
0.9 & 22 & 28 & 33 \\
1 & 20 & 25 & 29 \\
\hline
\end{tabular}

Table 2: Survival times with different values of $\varepsilon_{s}$ for different critical tumor masses. Decreasing the selfish resistance rate below 0.6 decreases the patient's survival time.

are minimized for the maximum resistance rate of 1 , and go higher as $\varepsilon_{s}$ is lowered. However, instead of a monotonic relationship, survival times are maximized for a resistance rate of 0.6 and go down again. This implies that treating a patient with resistance rate of 0.7 is dangerous, as exposing the selfish cancer cells to the immune system too much will lower life expectancy.

The explanation of this phenomenon comes from the phenotypes' competition for resources. Treatment of the selfish phenotype results in increased proliferation by the cooperative phenotype, making the tumor more aggressive without slowing its growth. This is in line with Proposition 3.4, as lowering the advantage of selfishness results in a cooperative tumor. For $\varepsilon_{s}=0$, the advantage of selfishness is nil, and lower than $r_{c} / r_{s}$, meaning that the tumor converges to the stable cooperative steady state, $x_{c 1}^{*}$. For all other displayed values of $\varepsilon_{s}$, the advantage of selfishness is higher than the ratio of birth rates, meaning that the tumor either converges to the stable selfish steady state $x_{s 1}^{*}$, or goes extinct. In the above cases, for $0.1 \leq \varepsilon_{s} \leq 0.6$, the tumor goes extinct (but not before potentially visiting the critical tumor mass), and for values $0.7 \leq \varepsilon_{s} \leq 1$, it converges to the stable selfish steady state.

Note that the ambiguous outcome of treatment effects in $\varepsilon_{s}$ is maintained for different configurations, even if $r_{c}<r_{s}$. In this case, the advantage of selfishness is always larger than $r_{c} / r_{s}$, hence the only ESS is the stable selfish steady state. However, if the immunosuppression parameter is large enough, a treatment of the selfish resistance rate may still worsen the patient's prognosis, as without enough selfish cells to compete with the cooperative cells, the highly aggressive cooperative phenotype gains a larger growth rate. Thus, even though steady-state tumor 
composition is preserved and treatment decreases steady-state tumor size, the survival time of the patient may still decrease.

\section{Conclusion}

We use game theory to model the dynamics of cancer cells and the immune system as a predatorprey system. The cancer cells exhibit two strategies. One confers resistance (safety) to the focal individual with no effect on the survival of others, while the second confers safety to both the individual and its neighbors. We refer to the former strategy as "selfish" because it only helps itself, and the latter as "cooperative" because it provides a public good. The model always exhibits three general outcomes: extinction of the cancer cells with some residual population of T-cells, the coexistence of one or the other cancer strategy with a population of T-cells, or the extinction of the T-cells with cancer cells going to their carrying capacity (this can only happen when the cancer cells are cooperative).

The model always results in a single pure-strategy ESS. If the two strategies share the same growth parameters, save for those associated with providing the public good, then the selfish strategy outcompetes the cooperative one. In this way, the game is a kind of prisoners dilemma embedded within a population model based on logistic growth and "predation" from the immune system. Others who have addressed the more general question of the evolution of cooperation, such as Axelrod and Axelrod (1984) have found that cooperation can prevail against selfishness if there are non-random interactions such as the clumping of individual by type in space (Nowak and May, 1992; Ale et al., 2013), or if cooperators can either withhold benefits from selfish individuals through conditional strategies such as Tit-for-Tat (Nowak and Sigmund, 1992), or otherwise punish defectors (Fudenberg and Maskin, 1986). Neither of these feature for evolving cooperation occur within our model. However, cooperation can be the ESS if the intrinsic growth rate of the cooperators is sufficiently higher than that of the selfish individuals. If the advantage of selfishness is higher than the ratio of reproduction rates, the tumor is selfish, if it is lower, the tumor is cooperative.

As a predator-prey model, our immune-cancer model has several properties and consequences. As victims of the T-cells, the cancer cells experience mortality that increases with the population size of T-cells. Furthermore, the T-cells exert mortality as a Type II-like functional response. This means that the cancer cells experience safety in numbers, regardless their type, and so their isocline is humped shaped as seen in Rosenzweig and MacArthur (1963). The upward sloping region of a cancer cell's isocline (in the state space of cancer cell and T-cell population 
sizes) represents an Allee effect (Taylor and Hastings, 2005) as safety in numbers more than compensates for intra-cancer cell competition.

The T-cell population is not a predator in the traditional sense. In our model they have a carrying capacity set by the innate immune system of the patient. This attribute is in line with predator-prey models where the predators receive a subsidy from outside of the system (Nevai) and Van Gorder, 2012). But, unlike these models, the T-cells gain no resources nor survival advantages from killing cancer cell. The growth rate of the T-cells does not increase with the mortality that they induce, rather, it is the overall population size of cancer cells that stimulates their growth rate. To incorporate this reality of T-cells, we let their carrying capacity increase with cancer cell population size. When the cancer cells are selfish, this produces a T-cell zeroisocline with a positive slope and a positive T-cell intercept. Combining the selfish cancer cell's and T-cell's isoclines produces up to two interior solutions. The first is an unstable extinction threshold, typical of many ecological models with an Allee effect. If the cancer population size drops too low they will go extinct and the cancer is cured. If the population size grows to a level above this threshold then a second, stable equilibrium is achieved (for some parameterizations this second interior equilibrium may yield a stable limit cycle) with a substantially higher cancer cell population and a higher T-cell population as well.

The interaction between the cooperative cancer cells and the immune system causes the model to deviate further from a standard predator-prey model. The immunosuppressive effect of the cooperative cancer cells means their presence exerts mortality on the T-cells - the cancer cells now have a predator-like effect on the T-cells even as they still enhance the T-cells' carrying capacity. The combined effects of the cooperative cancer cells on the T-cells means that the Tcell's zero-isocline can now either exhibit a hump (allowing for either two or even three interior equilibria), or it may be everywhere negatively sloped (allowing for either two interior equilibria or a single interior equilibrium). In this case, if the isoclines only permit a single interior equilibrium then it is a double extinction threshold, meaning that it separates the region where the T-cells drive the cancer cells extinct from the region where the cancer cells drive the T-cells extinct, meaning complete immunosuppression.

By considering the two immune evasion strategies our model reveals a more diverse array of transient dynamics and equilibria than the models of Robertson-Tessi et al. (2012) and Nakada et al. (2016). Furthermore, we have included the two ways that cancer cells might promote or inhibit T-cell persistence and recruitment in a more sophisticated and realistic fashion. Our two immune evasion strategies of selfish and cooperative mimic two broad classes of resistance strategies by cancer cells. In response to the immune system some cancer cells down-regulate the 
antigen that elicits T-cell attacks. This amounts to a form of crypticity that selfishly protects the focal cell while likely having little impact on the other cancer cells around it. On the other hand, cancer cells can also evolve the means to actually suppress T-cell recruitment and proliferation. This can be done by either directly signaling T-cells to cease functioning or by downregulating the cues that signal macrophages to stimulate T-cell proliferation (Gajewski et al., 2013).

Immunotherapy in our model acts through model parameters that enhance the lethal encounter rate of T-cells with cancer cells, reduce the advantage of selfishness (render crypticity ineffective), or decrease the immunosuppressive effect of cooperators (Ghirelli and Hagemann, 2013). These actions generally have positive therapeutic outcomes in terms of slowing the rate of tumor growth, increasing the extinction threshold below which the cancer will be cured, and decreasing the ultimate size of the tumor. If this size is below the lethal threshold for the patient then this may be a durable outcome, akin to adaptive therapy (Gatenby et al., 2009). However, by rendering crypticity ineffective, the tumor may shift from one dominated by selfish cells to one dominated by cooperative ones. As an unintended consequence, tumor size at the evolutionarily ESS may increase, and the patient's prognosis may be substantially worsened.

Our model can also consider other therapies (singly or in combination) where a cytotoxic drug or radiation therapy may influence the efficacy of the patient's natural immune system or additional immunotherapy. For instance, a cytotoxic drug may reduce the cancer cell's intrinsic growth rate by targeting cells with high proliferation rates. Or a targeted therapy may reduce the population size of cancer cells to below their extinction threshold. This becomes akin to the abscopal effect (Mole, 1953). A number of clinical examples exist where radiation therapy can induce a stronger immune response. If the cancer cell population is sufficiently depressed by radiation therapy, ours and other models suggest an extinction threshold where the immune system can now eradicate the residual cancer cell population.

A main takeaway of this paper is that in order to shrink, or fix tumor size through immunotherapy, the effects on tumor composition cannot be ignored. This is a well-known thesis in other aspects of cancer therapy, e.g. in the treatment of prostate cancer (You et al., 2017), but is yet to be adopted in immunotherapy. More specifically, our model identifies increasing immune efficiency and decreasing immunosuppression as viable treatment options. Both are shown to lower tumor burden and decrease the likelihood of cancer progression and metastasis via decreasing tumor size at the ESS, as well as to increase the patient's life expectancy. In most dynamic models of heterogeneous tumors - this paper's included - the quantitative effects of treatment with respect to tumor composition are analytically intractable. The same holds for life expectancy or most other proxies of the patient's prognosis. However, based on our work, 
a general qualitative statement can be formalized: treatment strategies that influence tumor composition are risky, whereas those that preserve composition are viable. Our findings merit more research aimed out identifying the risks and potential benefits of the former kind of treatment, and to validate our conclusions for the latter, whereas our proposed framework opens the possibility of integrating game theoretic concepts and ideas into the modeling of the interactions between heterogeneous tumors and the immune system.

\section{References}

Adam, J.A., and Maggelakis, S.A., 1989. Mathematical models of tumor growth. IV. Effects of a necrotic core. Mathematical Biosciences, 97(1), 121-136.

Ale, S.B., Brown, J.S., and Sullivan, A.T., 2013. Evolution of cooperation: combining kin selection and reciprocal altruism into matrix games with social dilemmas. PloS one, 8(5), p.e63761.

Axelrod, R., and Axelrod, R.M., 1984. The evolution of cooperation (Vol. 5145). Basic Books $(\mathrm{AZ})$.

Baar, M., Coquille, L., Mayer, H., Hölzel, M., Rogava, M., Tüting, T., and Bovier, A., 2016. A stochastic model for immunotherapy of cancer. Scientific Reports, 6, article nr. 24169.

Babbs, C.F., 2012. Predicting success or failure of immunotherapy for cancer: insights from a clinically applicable mathematical model. American Journal of Cancer Research, 2(2), 204-213.

De Pillis, L.G., and Radunskaya, A., 2001. A mathematical tumor model with immune resistance and drug therapy: an optimal control approach. Computational and Mathematical Methods in Medicine, 3(2), 79-100.

De Angelis, E., and Mesin, L., 2001. Modelling of the immune response: Conceptual frameworks and applications. Mathematical Models and Methods in Applied Sciences, 11(09), 1609-1630.

Dimberu, P.M., and Leonhardt, R.M., 2011. Cancer immunotherapy takes a multi-faceted approach to kick the immune system into gear. The Yale Journal of Biology and Medicine, 84(4), 371-380.

Dhodapkar, M.V., 2013. Personalized immune-interception of cancer and the battle of two adaptive systemswhen is the time right? Cancer Prevention Research, 6(3), 173-176. 
Eftimie, R., Bramson, J.L., and Earn, D.J.D., 2011. Interactions between the immune system and cancer: a brief review of non-spatial mathematical models. Bulletin of Mathematical Biology, 73(1), 2-32.

Foryś U., and Mokwa-Borkowska, A., 2005. Solid tumour growth analysis of necrotic core formation. Mathematical and Computer Modelling, 42(5-6), 593-600.

Fudenberg, D., and Maskin, E., 1986. The folk theorem in repeated games with discounting or with incomplete information. Econometrica: Journal of the Econometric Society, 533-554.

Gajewski, T.F., Schreiber, H., and Fu, Y.X., 2013. Innate and adaptive immune cells in the tumor microenvironment. Nature Immunology, 14(10), 1014-1022.

Gatenby, R.A., Silva, A.S., Gillies, R.J., and Frieden, B.R., 2009. Adaptive therapy. Cancer research, 69(11), 4894-4903.

Ghirelli, C., and Hagemann, T., 2013. Targeting immunosuppression for cancer therapy. The Journal of Clinical Investigation, 123(6), p.2355.

Goswami, S., Aparicio, A., and Subudhi, S.K., 2016. Immune checkpoint therapies in prostate cancer. Cancer Journal, 22(2), 117-120.

Hanahan, D., and Weinberg, R.A. 2011., Hallmarks of cancer: the next generation. Cell, 144(5), 646-674

Hicklin, D.J., Marincola, F.M., and Ferrone, S., 1999. HLA class I antigen downregulation in human cancers: T-cell immunotherapy revives an old story. Molecular Medicine Today, 5(4), $178-186$

Ibrahim-Hashim, A., Roberson-Tessi, M., Xu, L., Balagurunathan, Y., Wojtkowiak, J.W., Elkenawi, A.E., Enriquez-Navas, P.M., Russell, S., Kam, Y., Lloyd, M.C., Bui, M., Brown, J.S., Anderson, A.R.A., Gillies, R.J., and Gatenby, R.A., 2017. Defining cancer subpopulations by adaptive strategies rather than molecular properties provides novel insights into intratumoral evolution. Cancer Research 77, 2242-2254.

Johnsen, A.K., Templeton, D.J., Sy, M.S., and Harding, C.V., 1999. Deficiency of transporter for antigen presentation (TAP) in tumor cells allows evasion of immune surveillance and increases tumorigenesis. The Journal of Immunology, 163(8), 4224-4231. 
Kareva, I., and Berezovskaya, F., 2015. Cancer immunoediting: a process driven by metabolic competition as a predatorpreyshared resource type model. Journal of Theoretical Biology, 380, 463-472.

Maeurer, M.J., Gollin, S.M., Martin, D., Swaney, W., Bryant, J., Castelli, C., Robbins, P., Parmiani, G., Storkus, W.J., and Lotze, M.T., 1996. Tumor escape from immune recognition: lethal recurrent melanoma in a patient associated with downregulation of the peptide transporter protein TAP-1 and loss of expression of the immunodominant MART-1/Melan-A antigen. Journal of Clinical Investigation, 98(7), 1633-41.

Morgan, R.A., Dudley, M.E., Wunderlich, J.R., Hughes, M.S., Yang, J.C., Sherry, R.M., Royal, R.E., Topalian, S.L., Kammula, U.S., Restifo, N.P., Zheng, Z., Nahvi, A., de Vries, C.R., Rogers-Freezer, L.J., Mavroukakis, S.A., and Rosenberg, S.A., 2006. Cancer regression in patients after transfer of genetically engineered lymphocytes. Science, 314(5796), 126-129.

Nakada, N., Nagata, M., Takeuchi, Y., and Nakaoka, S., 2016. Mathematical modeling and analysis of combinational immune boost for tumor elimination. In AIP Conference Proceedings (Vol. 1723, No. 1, p. 020002). AIP Publishing.

Nani, F., and Freedman, H.I., 2000. A mathematical model of cancer treatment by immunotherapy. Mathematical Biosciences, 163(2), 159-199.

Nevai, A.L., and Van Gorder, R.A., 2012. Effect of resource subsidies on predatorprey population dynamics: a mathematical model. Journal of Biological Dynamics, 6(2), 891-922.

Nowak, M.A., and May, R.M., 1992. Evolutionary games and spatial chaos. Nature, 359(6398), 826-829.

Nowak, M.A., and Sigmund, K., 1992. Tit for tat in heterogeneous populations. Nature, $355(6357), 250-253$.

Mole, R.H., 1953. Whole body irradiation - radiobiology or medicine? The British Journal of Radiology, 26(305), 234-241.

Panetta, J.C., 1998. A mathematical model of drug resistance: heterogeneous tumors. Mathematical Biosciences, 147(1), 41-61.

Parcesepe, P., Giordano, G., Laudanna, C., Febbraro, A., and Pancione, M., 2016. Cancerassociated immune resistance and evasion of immune surveillance in colorectal cancer. Gastroenterology research and practice, vol. 2016, Article ID 6261721, 8 pages. 
Pardoll, D.M., 2012. The blockade of immune checkpoints in cancer immunotherapy. Nature Reviews Cancer, 12(4), 252-264.

Ribas, A., 2015. Adaptive immune resistance: How cancer protects from immune attack. Cancer Discovery, 5(9), 915919.

Robertson-Tessi, M., El-Kareh, A., and Goriely, A., 2012. A mathematical model of tumorimmune interactions. Journal of Theoretical Biology, 294, 56-73.

Rosenzweig, M.L., and MacArthur, R.H., 1963. Graphical representation and stability conditions of predator-prey interactions. The American Naturalist, 97(895), 209-223.

Seliger, B., Maeurer, M. J., and Ferrone, S. 1997. TAP off - tumors on. Immunology today, 18(6), 292-299.

Sharma, P., Hu-Lieskovan, S., Wargo, J. A., and Ribas, A., 2017. Primary, adaptive, and acquired resistance to cancer immunotherapy. Cell, 168(4), 707-723.

Smyth, M.J., Dunn, G.P., and Schreiber, R.D., 2006. Cancer immunosurveillance and immunoediting: the roles of immunity in suppressing tumor development and shaping tumor immunogenicity. Advances in Immunology, 90, 1-50.

Stein, J., 1981. Prey-predator-protector model for cancer. IEEE Transactions on Biomedical Engineering, (7), 544-549.

Stewart, T.J., and Smyth, M.J., 2011. Improving cancer immunotherapy by targeting tumorinduced immunosuppression. Cancer Metastasis Rev, 30(1), 125-140.

Sun, X., Bao, J., and Shao, Y., 2016. Mathematical modeling of therapy-induced cancer drug resistance: connecting cancer mechanisms to population survival rates. Scientific Reports, 6 .

Taylor, C.M., and Hastings, A., 2005. Allee effects in biological invasions. Ecology Letters, 8(8), 895-908.

Tobin, P.C., Berec, L., and Liebhold, A.M., 2011. Exploiting Allee effects for managing biological invasions. Ecology Letters, 14(6), 615-624.

Tomasetti, C., and Levy, D., 2010. An elementary approach to modeling drug resistance in cancer. Mathematical Biosciences and Engineering: MBE, 7(4), 905. 
Vinay, D.S., Ryan, E.P., Pawelec, G., Talib, W.H., Stagg, J., Elkord, E., Lichtor, T., Decker, W.K., Whelan, R.L., Kumara, H.S., and Signori, E., 2015. Immune evasion in cancer: Mechanistic basis and therapeutic strategies. In Seminars in Cancer Biology 35, S185-S198. Academic Press.

Wu, A.A., Drake, V., Huang, H.S., Chiu, S., and Zheng, L., 2015. Reprogramming the tumor microenvironment: tumor-induced immunosuppressive factors paralyze T cells. Oncoimmunology, 4(7), p. e1016700.

You, L., Brown, J.S., Thuijsman, F., Cunningham, J.J., Gatenby, R.A., Zhang, J., and Staňková, K., 2017. Spatial vs. non-spatial eco-evolutionary dynamics in a tumor growth model. Journal of Theoretical Biology, 435, 78-97.

\section{Appendix}

Proposition 3.6. Let $D_{s}>0$. If $x_{s 1}^{*}>\frac{K^{2}}{4}$, then $\left(x_{s 1}^{*}, y_{s 1}^{*}, 0\right)$ is linearly stable in $x$ and $y$.

Proof. The system is as follows.

$$
\begin{aligned}
& \dot{x}=r_{s}\left(1-\frac{\sqrt{x}}{K}\right) x-\gamma\left(1-\varepsilon_{s}\right) \sqrt{x} y=F(x, y), \\
& \dot{y}=r_{i}\left(1-\frac{y}{L+a \sqrt{x}}\right) y=G(x, y) .
\end{aligned}
$$

Consider the Jacobian of the problem.

$$
J\left(x_{s 1}^{*}, y_{s 1}^{*}\right)=\left(\begin{array}{ll}
\frac{\partial F(x, y)}{\partial x} & \frac{\partial F(x, y)}{\partial y} \\
\frac{\partial G(x, y)}{\partial x} & \frac{\partial G(x, y)}{\partial y}
\end{array}\right)\left(x_{s 1}^{*}, y_{s 1}^{*}\right) .
$$

The elements are as follows. We have $1, \frac{\partial F(x, y)}{\partial x}\left(x_{s 1}^{*}, y_{s 1}^{*}\right)<0$. We can show this by taking

$$
\frac{\partial F(x, y)}{\partial x}=r_{s}\left(1-\frac{3 \sqrt{x}}{2 K}\right)-\frac{y}{2 \sqrt{x}} \gamma\left(1-\varepsilon_{s}\right) .
$$

Note that since $\dot{x}=0$ at the steady state, we have $r_{s}\left(1-\frac{\sqrt{x_{s 1}^{*}}}{K}\right)=\gamma\left(1-\varepsilon_{s}\right) \frac{y_{s 1}^{*}}{\sqrt{x_{s 1}^{*}}}$. Thus, substituting gives

$$
\frac{\partial F(x, y)}{\partial x}\left(x_{s 1}^{*}, y_{s 1}^{*}\right)=r_{s}\left(\frac{1}{2}-\frac{\sqrt{x_{s 1}^{*}}}{K}\right)<0
$$

by using $\sqrt{x_{s 1}^{*}}>K / 2$.

$2, \frac{\partial F(x, y)}{\partial y}\left(x_{s 1}^{*}, y_{s 1}^{*}\right)<0$. This is shown by simply taking

$$
\frac{\partial F(x, y)}{\partial y}=-\gamma\left(1-\varepsilon_{s}\right) \sqrt{x}<0
$$


for all $x>0$.

$3, \frac{\partial G(x, y)}{\partial x}\left(x_{s 1}^{*}, y_{s 1}^{*}\right)>0$. This is shown by taking

$$
\frac{\partial G(x, y)}{\partial x}=r_{i} \frac{a}{2 \sqrt{x}} \frac{y^{2}}{(L+a \sqrt{x})^{2}}>0,
$$

for all $x, y>0$.

$4, \frac{\partial G(x, y)}{\partial y}\left(x_{s 1}^{*}, y_{s 1}^{*}\right)<0$. This is shown by taking

$$
\frac{\partial G(x, y)}{\partial y}=r_{i}-\frac{2 y}{L+a \sqrt{x}}
$$

At the steady state we have $y_{s 1}^{*}=L+a \sqrt{x_{s 1}^{*}}$, thus $\frac{\partial G(x, y)}{\partial y}\left(x_{s 1}^{*}, y_{s 1}^{*}\right)=r_{i}-2 r_{i}=-r_{i}<0$.

So, qualitatively, we have

$$
J\left(x_{s 1}^{*}, y_{s 1}^{*}\right)=\left(\begin{array}{cc}
- & - \\
+ & -
\end{array}\right)
$$

meaning that $\operatorname{Tr}\left(J\left(x_{s 1}^{*}, y_{s 1}^{*}\right)\right)<0$, and $\operatorname{Det}\left(J\left(x_{s 1}^{*}, y_{s 1}^{*}\right)\right)>0$, thus the steady state $\left(x_{s 1}^{*}, y_{s 1}^{*}\right)$ is stable for deviations in $x$ and $y$.

Lemma 5.1. For $\left(x_{c 1}^{*}, y_{c 1}^{*}, 1\right)$ it holds that

$$
\frac{x_{c 1}^{*}}{K\left(L+\frac{a d}{r_{i}} x_{c 1}^{*}\right)}>\frac{\gamma\left(1-\varepsilon_{c}\right)}{r_{c}} .
$$

Proof. At $\left(x_{c 1}^{*}, y_{c 1}^{*}, 1\right)$, the zero isocline of $y$ intersects that of $x$ from below. Hence, the derivative of the former, evaluated at the steady state, is greater:

$$
\sqrt{x_{c 1}^{*}}\left(\frac{1}{2}-\frac{\sqrt{x_{c 1}^{*}}}{K}\right) \frac{r_{c}}{\gamma\left(1-\varepsilon_{c}\right)}<\frac{a}{2} \sqrt{x_{c 1}^{*}}-\frac{\delta}{2 r_{i}} L \sqrt{x_{c 1}^{*}}-a \frac{\delta}{r_{i}} x_{c 1}^{*} .
$$

At the same time, since the point $\left(x_{c 1}^{*}, y_{c 1}^{*}\right)$ lies upon both isoclines we have

$$
y_{c 1}^{*}=\sqrt{x_{c 1}^{*}}\left(1-\frac{\sqrt{x_{c 1}^{*}}}{K}\right) \frac{r_{c}}{\gamma\left(1-\varepsilon_{c}\right)}=L+a \sqrt{x_{c 1}^{*}}-\frac{\delta}{r_{i}} L \sqrt{x_{c 1}^{*}}-a \frac{\delta}{r_{i}} x_{c 1}^{*} .
$$

Hence, we get

$$
\sqrt{x_{c 1}^{*}}\left(1-\frac{\sqrt{x_{c 1}^{*}}}{K}\right) \frac{r_{c}}{\gamma\left(1-\varepsilon_{c}\right)}-L+a \frac{\delta}{r_{i}} x_{c 1}^{*}=a \sqrt{x_{c 1}^{*}}-\frac{\delta}{r_{i}} L \sqrt{x_{c 1}^{*}} .
$$

Applying 8 gives

$$
\sqrt{x_{c 1}^{*}}\left(1-\frac{\sqrt{x_{c 1}^{*}}}{K}\right) \frac{r_{c}}{\gamma\left(1-\varepsilon_{c}\right)}-L+a \frac{\delta}{r_{i}} x_{c 1}^{*}>2 \sqrt{x_{c 1}^{*}}\left(\frac{1}{2}-\frac{\sqrt{x_{c 1}^{*}}}{K}\right) \frac{r_{c}}{\gamma\left(1-\varepsilon_{c}\right)}+2 a \frac{\delta}{r_{i}} x_{c 1}^{*} .
$$

After rearranging we have

$$
\frac{x_{c 1}^{*}}{K} \frac{r_{c}}{\gamma\left(1-\varepsilon_{c}\right)}-L-a \frac{\delta}{r_{i}} x_{c 1}^{*}>0 .
$$


A final rearrangement gives

$$
\frac{x_{c 1}^{*}}{K\left(L+\frac{a d}{r_{i}} x_{c 1}^{*}\right)}>\frac{\gamma\left(1-\varepsilon_{c}\right)}{r_{c}}
$$

as stated.

Proposition 3.11 Let $D_{c}>0$. If $x_{c 1}^{*}>\frac{K^{2}}{4}$, then $\left(x_{c 1}^{*}, y_{c 1}^{*}, 1\right)$ is linearly stable in $x$ and $y$. Proof. The system is as follows.

$$
\begin{aligned}
& \dot{x}=r_{c}\left(1-\frac{\sqrt{x}}{K}\right) x-\gamma\left(1-\varepsilon_{c}\right) \sqrt{x} y=F(x, y), \\
& \dot{y}=r_{i}\left(1-\frac{y}{L+a \sqrt{x}}\right) y-\delta \sqrt{x} y=G(x, y) .
\end{aligned}
$$

Again, consider the Jacobian of the problem.

$$
J\left(x_{c 1}^{*}, y_{c 1}^{*}\right)=\left(\begin{array}{ll}
\frac{\partial F(x, y)}{\partial x} & \frac{\partial F(x, y)}{\partial y} \\
\frac{\partial G(x, y)}{\partial x} & \frac{\partial G(x, y)}{\partial y}
\end{array}\right)\left(x_{c 1}^{*}, y_{c 1}^{*}\right) .
$$

As before, we have $1, \frac{\partial F(x, y)}{\partial x}\left(x_{c 1}^{*}, y_{c 1}^{*}\right)=r_{c}\left(1-\frac{3 \sqrt{x_{c 1}^{*}}}{2 K}\right)-\frac{y_{c 1}^{*}}{2 \sqrt{x_{c 1}^{*}}} \gamma\left(1-\varepsilon_{c}\right)=r_{c}\left(\frac{1}{2}-\frac{\sqrt{x_{c 1}^{*}}}{K}\right)$.

$2, \frac{\partial F(x, y)}{\partial y}\left(x_{c 1}^{*}, y_{c 1}^{*}\right)=-\gamma\left(1-\varepsilon_{c}\right) \sqrt{x_{c 1}^{*}}$. Since $\left(x_{c 1}^{*}, y_{c 1}^{*}\right)$ is on the zero-isocline of $x$ we have $\gamma\left(1-\varepsilon_{c}\right) \sqrt{x_{c 1}^{*}} y_{c 1}^{*}=r_{c}\left(1-\frac{\sqrt{x_{c 1}^{*}}}{K}\right) x_{c 1}^{*}$, hence

$$
\frac{\partial F(x, y)}{\partial y}\left(x_{c 1}^{*}, y_{c 1}^{*}\right)=-r_{c}\left(1-\frac{\sqrt{x_{c 1}^{*}}}{K}\right) \frac{x_{c 1}^{*}}{y_{c 1}^{*}}
$$

$3, \frac{\partial G(x, y)}{\partial x}\left(x_{c 1}^{*}, y_{c 1}^{*}\right)=r_{i} \frac{a}{2 \sqrt{x_{c 1}^{*}}} \frac{y_{c 1}^{2}}{\left(L+a \sqrt{x_{c 1}^{*}}\right)^{2}}-\frac{\delta}{2 \sqrt{x_{c 1}^{*}}} y_{c 1}^{*}$. Since the steady state is on the zeroisocline of $y$ we have $r_{i}\left(1-\frac{y_{c 1}^{*}}{L+a \sqrt{x_{c 1}^{*}}}\right)=\delta \sqrt{x_{c 1}^{*}}$, hence

$$
\frac{\partial G(x, y)}{\partial x}\left(x_{c 1}^{*}, y_{c 1}^{*}\right)=\frac{1}{2} r_{i}\left(a \sqrt{x_{c 1}^{*}} \frac{y_{c 1}^{*}}{\left(L+a \sqrt{x_{c 1}^{*}}\right)^{2}}+\frac{y_{c 1}^{*}}{L+a \sqrt{x}_{c 1}}-1\right) \frac{y_{c 1}^{*}}{x_{c 1}^{*}} .
$$
gives

$4, \frac{\partial G(x, y)}{\partial y}=r_{i}\left(1-\frac{2 y}{L+a \sqrt{x}}\right)-\delta \sqrt{x}$. Using the steady-state condition $r_{i}\left(1-\frac{y_{c 1}^{*}}{L+a \sqrt{x_{c 1}^{*}}}\right)=\delta \sqrt{x_{c 1}^{*}}$

$$
\frac{\partial G(x, y)}{\partial y}\left(x_{c 1}^{*}, y_{c 1}^{*}\right)=-r_{i} \frac{y_{c 1}^{*}}{L+a \sqrt{x_{c 1}^{*}}} .
$$

As before, $\operatorname{Tr}\left(J\left(x_{c 1}^{*}, y_{c 1}^{*}\right)\right)<0$, for $x_{c 1}^{*}>K / 4$. For the determinant, we calculate

$$
\begin{aligned}
& \frac{\partial F(x, y)}{\partial x}\left(x_{c 1}^{*}, y_{c 1}^{*}\right) \frac{\partial G(x, y)}{\partial y}\left(x_{c 1}^{*}, y_{c 1}^{*}\right)=-r_{c} r_{i}\left(\frac{1}{2}-\frac{\sqrt{x_{c 1}^{*}}}{K}\right) \frac{y_{c 1}^{*}}{L+a \sqrt{x_{c 1}^{*}}}, \\
& \frac{\partial F(x, y)}{\partial y}\left(x_{c 1}^{*}, y_{c 1}^{*}\right) \frac{\partial G(x, y)}{\partial x}\left(x_{c 1}^{*}, y_{c 1}^{*}\right)=-\frac{1}{2} r_{c} r_{i}\left(1-\frac{\sqrt{x_{c 1}^{*}}}{K}\right)\left(a \sqrt{x_{c 1}^{*}} \frac{y_{c 1}^{*}}{\left(L+a \sqrt{x_{c 1}^{*}}\right)^{2}}+\frac{y_{c 1}^{*}}{L+a \sqrt{x}_{c 1}}-1\right) .
\end{aligned}
$$


Therefore, the determinant is as follows:

$$
\begin{gathered}
\operatorname{Det}\left(J\left(x_{c 1}^{*}, y_{c 1}^{*}\right)\right)=\frac{1}{2} r_{c} r_{i}\left(\frac{\sqrt{x_{c 1}^{*}}}{K}-1-\frac{\sqrt{x_{c 1}^{*}}}{K} \frac{a \sqrt{x_{c 1}^{*}} y_{c 1}^{*}}{\left(L+a \sqrt{x_{c 1}^{*}}\right)^{2}}+\frac{a \sqrt{x_{c 1}^{*}} y_{c 1}^{*}}{\left(L+a \sqrt{x_{c 1}^{*}}\right)^{2}}+\frac{\sqrt{x_{c 1}^{*}}}{K} \frac{y_{c 1}^{*}}{L+a \sqrt{x}}\right), \\
\left.\operatorname{Det}\left(J\left(x_{c 1}^{*}, y_{c 1}^{*}\right)\right)=\frac{1}{2} r_{c} r_{i}\left(\left(1-\frac{\sqrt{x_{c 1}^{*}}}{K}\right)\left(\frac{a \sqrt{x_{c 1}^{*}} y_{c 1}^{*}}{\left(L+a \sqrt{x_{c 1}^{*}}\right)^{2}}-1\right)\right)+\frac{\sqrt{x_{c 1}^{*}}}{K} \frac{y_{c 1}^{*}}{L+a \sqrt{x}_{c 1}}\right) .
\end{gathered}
$$

We use the fact that the steady state lies along the zero-isocline of $x$ and substitute $y_{c 1}^{*}=$ $\sqrt{x_{c 1}^{*}}\left(1-\frac{\sqrt{x_{c 1}^{*}}}{K}\right) \frac{r_{c}}{\gamma\left(1-\varepsilon_{c}\right)}$ into the last bracketed term to get

$$
\operatorname{Det}\left(J\left(x_{c 1}^{*}, y_{c 1}^{*}\right)\right)=\frac{1}{2} r_{c} r_{i}\left(1-\frac{\sqrt{x_{c 1}^{*}}}{K}\right)\left(\frac{x_{c 1}^{*}}{K} \frac{r_{c}}{\gamma\left(1-\varepsilon_{c}\right)} \frac{1}{L+a \sqrt{x_{c 1}^{*}}}+\frac{y_{c 1}^{*} a \sqrt{x_{c 1}^{*}}}{\left(L+a \sqrt{x_{c 1}^{*}}\right)^{2}}-1\right) .
$$

Now we use the zero-isocline of $y$ and substitute $y_{c 1}^{*}=\left(L+a \sqrt{x_{c 1}^{*}}\right)\left(1-\frac{\delta}{r_{i}} \sqrt{x_{c 1}^{*}}\right)$, which gives

$$
\operatorname{Det}\left(J\left(x_{c 1}^{*}, y_{c 1}^{*}\right)\right)=\frac{1}{2} r_{c} r_{i}\left(1-\frac{\sqrt{x_{c 1}^{*}}}{K}\right)\left(\frac{x_{c 1}^{*}}{K} \frac{r_{c}}{\gamma\left(1-\varepsilon_{c}\right)} \frac{1}{L+a \sqrt{x_{c 1}^{*}}}+a \sqrt{x_{c 1}^{*}}\left(1-\frac{\delta}{r_{i}} \sqrt{x_{c 1}^{*}}\right) \frac{1}{L+a \sqrt{x_{c 1}^{*}}}-1\right) .
$$

Therefore, $\operatorname{Det}\left(J\left(x_{c 1}^{*}, y_{c 1}^{*}\right)\right)>0$ if and only if we have

$$
\frac{x_{c 1}^{*}}{K} \frac{r_{c}}{\gamma\left(1-\varepsilon_{c}\right)}+a \sqrt{x_{c 1}^{*}}\left(1-\frac{\delta}{r_{i}} \sqrt{x_{c 1}^{*}}\right)>L+a \sqrt{x_{c 1}^{*}} .
$$

Rearranging gives the condition as

$$
\frac{x_{c 1}^{*}}{K\left(L+\frac{a d}{r_{i}} x_{c 1}^{*}\right)}>\frac{\gamma\left(1-\varepsilon_{c}\right)}{r_{c}} .
$$

By Lemma 5.1, this holds, hence the proof is complete. 